\title{
Quantifying non-Gaussianity for quantum information
}

\author{
Marco G. Genoni* \\ Consorzio Nazionale Interuniversitario per le Scienze Fisiche della Materia, UdR Milano, I-20133 Milano, Italy \\ Matteo G. A. Paris ${ }^{\dagger}$ \\ Dipartimento di Fisica, Università degli Studi di Milano, I-20133 Milano, Italy
}

(Received 10 September 2010; published 30 November 2010)

\begin{abstract}
We address the quantification of non-Gaussianity $(\mathrm{nG})$ of states and operations in continuous-variable systems and its use in quantum information. We start by illustrating in detail the properties and the relationships of two recently proposed measures of $\mathrm{nG}$ based on the Hilbert-Schmidt distance and the quantum relative entropy (QRE) between the state under examination and a reference Gaussian state. We then evaluate the non-Gaussianities of several families of non-Gaussian quantum states and show that the two measures have the same basic properties and also share the same qualitative behavior in most of the examples taken into account. However, we also show that they introduce a different relation of order; that is, they are not strictly monotone to each other. We exploit the $\mathrm{nG}$ measures for states in order to introduce a measure of $\mathrm{nG}$ for quantum operations, to assess Gaussification and de-Gaussification protocols, and to investigate in detail the role played by $\mathrm{nG}$ in entanglement-distillation protocols. Besides, we exploit the QRE-based $\mathrm{nG}$ measure to provide different insight on the extremality of Gaussian states for some entropic quantities such as conditional entropy, mutual information, and the Holevo bound. We also deal with parameter estimation and present a theorem connecting the QRE nG to the quantum Fisher information. Finally, since evaluation of the QRE nG measure requires the knowledge of the full density matrix, we derive some experimentally friendly lower bounds to $\mathrm{nG}$ for some classes of states and by considering the possibility of performing on the states only certain efficient or inefficient measurements.
\end{abstract}

DOI: 10.1103/PhysRevA.82.052341

PACS number(s): 03.67.-a, 03.65.Ud, 42.50.Dv

\section{INTRODUCTION}

In the recent years we have witnessed a big effort in the theoretical and experimental investigation of continuousvariable (CV) quantum information. Gaussian states are experimentally produced with an high degree of control, especially in quantum optics, and Gaussian measurements may be effectively implemented in different settings. Besides, despite belonging to an infinite-dimensional Hilbert space, Gaussian states are easy to handle from a theoretical point of view, being fully described by the first and second moments of the canonical operators [1-3]. The remarkable role of Gaussian states has been highlighted in [4], where it has been proved that they are extremal at fixed covariance matrix for several relevant quantities as channel capacities and entanglement measures and also in the framework of CV quantum key distribution in [5-7], where it has been shown that Gaussian attacks are optimal against all individual and collective eavesdropping strategies. For these reasons, Gaussian states played a prominent role in the development of $\mathrm{CV}$ quantum information and, as a matter of fact, most of the protocols designed for finite-dimensional Hilbert spaces have been first translated in the CV setting for Gaussian states [8].

In the recent years, however, it has been realized that there are situations wherein non-Gaussianity $(\mathrm{nG})$ in the form of non-Gaussian states or non-Gaussian operations is either required or desirable to achieve some relevant tasks in quantum information processing. As for example, it is known that $\mathrm{nG}$ is crucial for the realization of entanglement distillation [9-11],

\footnotetext{
*marco.genoni@fisica.unimi.it

†matteo.paris@ fisica.unimi.it
}

quantum error correction [12], and cluster-state quantum computation [13,14]. Besides, a non-Gaussian measurement and/or non-Gaussian states are crucial for observing violations of loophole-free Bell tests with CVs [15-24]. In addition, improvement of quantum teleportation and quantum cloning of coherent states can be obtained by using, respectively, non-Gaussian states or non-Gaussian operations [25-28]. In turn, bipartite Gaussian states have minimum entanglement for given second moments and this influences their performances in quantum information protocols. Non-Gaussian operations also find application in noiseless amplification [29,30] obtained in conditional fashion, whereas non-Gaussian states have been proven useful in improving parameter estimation in quantum optics [31,32]. The current state of the art is schematically depicted in Fig. 1.

For the reasons outlined previously, several protocols have been designed theoretically [33,34] and experimentally realized [35-45] to produce single-mode or two-mode nonGaussian states in different physical settings and, in particular, to perform squeezing purification [46] and CV entanglement distillation [40,47-49]. Basically, they may be divided into two main categories: those based on nonlinear interaction of order higher than two [50,51], as for example the Kerr effect [52-54], and those based on conditional measurements. Indeed, the nonlinear dynamics induced by conditional measurements has been analyzed for a large variety of schemes [55-69], including, besides photon addition and subtraction schemes, optical state truncation of coherent states [58], state filtering by active cavities [59,60], synthesis of arbitrary unitary operators [61], and generation of optical qubit by conditional interferometry [62]. Conditional state generation has been achieved in the low-energy regime $[45,49,70]$ by using single-photon detectors and also in the mesoscopic domain 


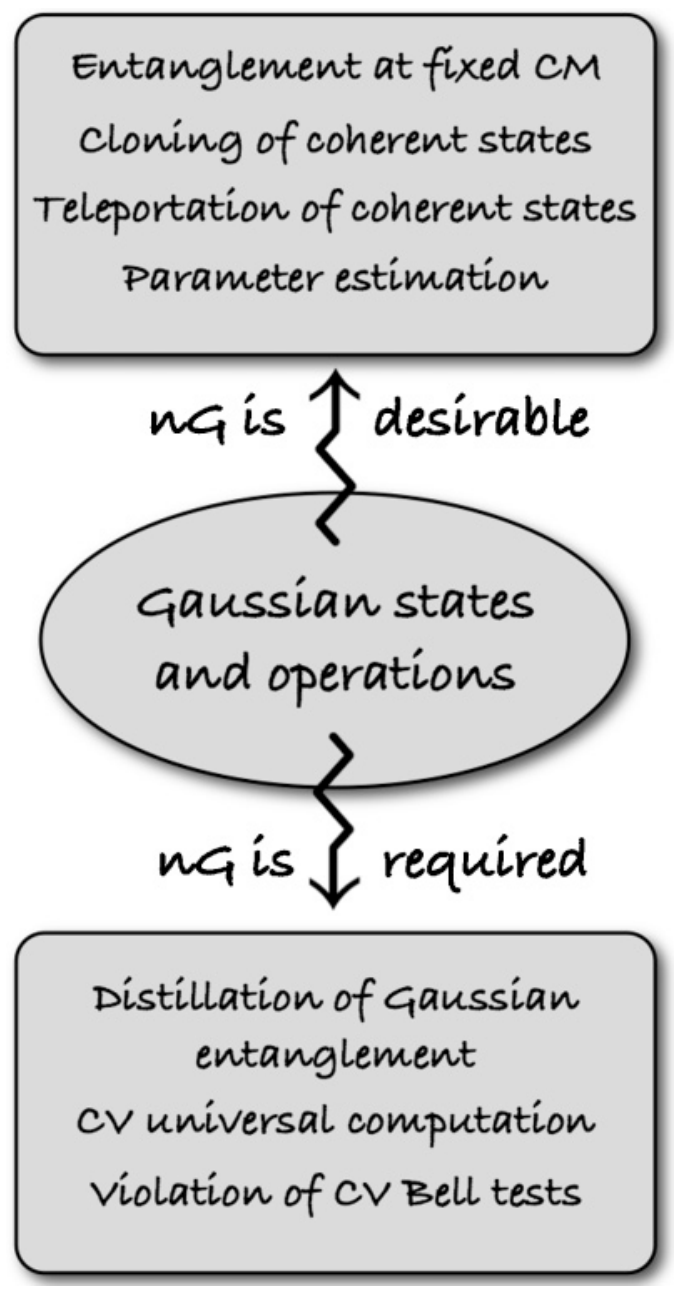

FIG. 1. The departure from the Gaussian world is required to achieve specific tasks such as the distillation of Gaussian entanglement, universal quantum computation with Gaussian cluster states, and the violation of loophole-free Bell tests with CVs (bottom box) and lead to an increase of entanglement at fixed covariance matrix, with improvement of relevant protocols such as teleportation and cloning of coherent states and parameter estimation of both unitary and lossy channels (top box).

[71-73]. Realizations of non-Gaussian states have been also reported in optical cavities [74] and in superconducting circuits [75].

Being recognized as a resource for $\mathrm{CV}$ quantum information, the need of quantifying the non-Gaussian character of states and operations naturally arises and different measures of $n G$ have been proposed [76-78]. These measures have been used to assess the role of $\mathrm{nG}$ in different quantum information and communication tasks such as teleportation [79], quantum estimation [31], experimental entanglement quantification [80], and entanglement transfer between CV states and qubits [81,82]. In [83] the relationship between $\mathrm{nG}$ and the Hudson's theorem [84] have been studied, obtaining at fixed purity an upper bound for non-Gaussian states having a positive Wigner function, while in [85] nG bounded uncertainty relations are derived. The entropic measure proposed in [77] has been used to quantify exactly the $\mathrm{nG}$ of experimentally produced photon-added coherent states and a lower bound has been evaluated experimentally in [86] for conditional states obtained via an inefficient photodetection on classically correlated thermal beams.

In this paper, we address in detail the quantification of $\mathrm{nG}$ of states and operations in CV systems and analyze its use in quantum information. At first, we review the two measures proposed in [76] and [77] by studying in more detail their properties and their relationships and then exploit them to assess some relevant Gaussification and de-Gaussification protocols, and to investigate the role of $\mathrm{nG}$ in entanglement distillation, quantum communication, and quantum estimation.

The paper is structured as follows: In the next section we briefly review some topics on the quantification of $\mathrm{nG}$ of a classical probability (density) distribution. In Sec. III we introduce notation and give the definition of Gaussian states along with their properties, while in Sec. IV we review the two quantum measures of $\mathrm{nG}$, proving their properties and highlighting the relationships between them. In Sec. $\mathrm{V}$ we evaluate the $\mathrm{nG}$ measures for some relevant non-Gaussian states, comparing them and observing if and when they give the same order relation. In Sec. VI we employ the two measures of $\mathrm{nG}$ to address the Gaussification process due to the interaction of the system with a bath of harmonic oscillators in the vacuum state (i.e., dissipation a zero temperature) and the de-Gaussification process due either to phase-diffusion or to self-Kerr interaction. In Sec. VII we study the role of $\mathrm{nG}$ in two paradigmatic examples of entanglement-distillation protocols, while in Sec. VIII we show how the amount of $\mathrm{nG}$ is related to some entropicinformational quantities as the Holevo bound, conditional entropy, and mutual information. In Sec. IX we deal with parameter estimation and present a theorem relating the $\mathrm{nG}$ and the quantum Fisher information. Finally, in Sec. X we address the experimental evaluation of $\mathrm{nG}$ in situations where state tomography is not available and present some experimentally friendly bounds for the estimation of $\mathrm{nG}$ of some classes of states. Section XI closes the paper with some concluding remarks.

\section{NON-GAUSSIANITY OF A CLASSICAL PROBABILITY DISTRIBUTION}

According to the central limit theorem, the Gaussian distribution is ubiquitous in the description of natural phenomena. In turn, deviations from the Gaussian behavior are often the sign that an interesting phenomenon occurs [87-92], and thus considerable attention has been devoted to the detection and quantification of $\mathrm{nG}$ of a classical distribution. Basically, there are two main approaches. The first one is based on the evaluation of higher moments of the distribution, in particular, the third and the fourth central moments, to assess skewness and kurtosis of the distribution in comparison to those of a Gaussian one. The second approach is based on the evaluation of the Shannon entropy of the distribution, upon the fact that Gaussian distributions maximize it at fixed variance. More recently, it turned out that $\mathrm{nG}$ is relevant in the framework of independent component analysis (ICA) [93]. ICA is a method developed in the past decades in which the goal is to find a linear representation of non-Gaussian data so that the components are statistically independent. In this method 
the solution is obtained by the maximization of the $\mathrm{nG}$ of the components and thus, to accomplish this goal, different measures of $\mathrm{nG}$ have been proposed.

Let us consider a scalar-valued random variable $Y$ with a probability density function $p(y):=P(Y=y)$. Its $k$ th central moments are defined as

$$
E\left[(Y-\mu)^{k}\right]=\int_{-\infty}^{+\infty} d y(y-\mu)^{k} p(y),
$$

where

$$
\mu=\int_{-\infty}^{+\infty} d y \text { y } p(y)
$$

is the mean value of the distribution. We say that $Y$ is Gaussian distributed if its probability density function is a Gaussian function, that is,

$$
p(y)=\frac{1}{\sqrt{2 \pi \sigma^{2}}} \exp \left\{-\frac{y-\mu}{2 \sigma^{2}}\right\},
$$

where $\sigma^{2}=E\left[(Y-\mu)^{2}\right]$ is the second moment of the distribution, called variance. In the following we present the definition of two measures of $\mathrm{nG}$ for a classical scalar-valued random variable.

\section{A. Kurtosis}

The fist considered measure of $\mathrm{nG}$ has been the kurtosis, that is, the fourth-order cumulant defined by the formula

$$
K(Y)=E\left[(Y-\mu)^{4}\right]-3 \sigma^{2} .
$$

$K(Y)$ is zero for a Gaussian random variable, while for most (but not all) non-Gaussian random variables it takes values different from zero. kurtosis can be positive or negative. Random variables that have negative kurtosis are called sub-Gaussian and are characterized by a probability density function with heavy tails. On the other hand, the ones with positive kurtosis are called super-Gaussian and they have typically a "flat" distribution (constant near the mean value and very small for "distant" values of the variable). Anyway, typically, the $\mathrm{nG}$ is measured by the absolute value or the square of the kurtosis. While it is relatively simple to evaluate it if the probability density function is known, kurtosis presents some drawbacks when its value has to be estimated from a measured sample. Indeed, it can be very sensitive to outliers and its value may strongly depend on only a few observations in the tails of the distribution. For these reasons kurtosis is not considered a robust measure of $\mathrm{nG}$.

\section{B. Negentropy}

Given a discrete random variable $X=\left\{x_{1}, \ldots, x_{n}\right\}$ with a probability distribution $p\left(x_{i}\right)=P\left(X=x_{i}\right)$, we can define its (Shannon) entropy as

$$
H(X)=-\sum_{i} p\left(x_{i}\right) \ln p\left(x_{i}\right) .
$$

This definition can be generalized for a continuous-valued random variable $Y$, in which case it is called differential entropy:

$$
\mathrm{H}(Y)=-\int d y p(y) \ln p(y) .
$$

A fundamental result of information theory states that at fixed variance, Gaussian variables have the largest entropy. Following this result, one may define a measure of $\mathrm{nG}$, called negentropy, as

$$
N(Y)=\mathrm{H}(G)-\mathrm{H}(Y),
$$

where $G$ is the Gaussian random variable with the same variance of $Y$. Due to the previously mentioned result, negentropy is always non-negative and it is equal to zero only for Gaussian random variables. Negentropy is thus well justified by statistical theory but its computation is typically very difficult. However, simpler approximations based on evaluations of moments of the random variable have been introduced and used for ICA purposes [94]. In the following we see that one of the two quantum measures that are analyzed, even if defined starting from an another quantity, result in the quantum analog of the negentropy here presented.

\section{QUANTUM GAUSSIAN STATES}

For concreteness, we use here the quantum optical terminology of modes carrying photons, but our approach may be equally applied to any bosonic CV system. Let us consider a system of $n$ modes described by mode operators $a_{k}, k=$ $1, \ldots, n$, satisfying the commutation relations $\left[a_{k}, a_{j}^{\dagger}\right]=\delta_{k j}$. A quantum state $\varrho$ of the $n$ modes is fully described by its characteristic function [95],

$$
\chi[\varrho](\lambda)=\operatorname{Tr}[\varrho D(\lambda)],
$$

where $D(\lambda)=\bigotimes_{k=1}^{n} D_{k}\left(\lambda_{k}\right)$ is the $n$-mode displacement operator, with $\lambda=\left(\lambda_{1}, \ldots, \lambda_{n}\right)^{T}, \lambda_{k} \in \mathbb{C}$, and where

$$
D_{k}\left(\lambda_{k}\right)=\exp \left\{\lambda_{k} a_{k}^{\dagger}-\lambda_{k}^{*} a_{k}\right\}
$$

is the single-mode displacement operator. Analogously, quantum states can be fully described by the Wigner function, that is, the Fourier transform of the characteristic function:

$$
W[\varrho](\alpha)=\int \frac{d^{2 n} \lambda}{\pi^{2 n}} e^{\lambda^{*} \alpha+\alpha^{*} \lambda} \chi[\varrho](\lambda) .
$$

The canonical operators are given by

$$
\begin{aligned}
& q_{k}=\frac{1}{\sqrt{2}}\left(a_{k}+a_{k}^{\dagger}\right), \\
& p_{k}=\frac{1}{i \sqrt{2}}\left(a_{k}-a_{k}^{\dagger}\right),
\end{aligned}
$$

with commutation relations given by $\left[q_{j}, p_{k}\right]=i \delta_{j k}$. Upon introducing the real vector $\boldsymbol{R}=\left(q_{1}, p_{1}, \ldots, q_{n}, p_{n}\right)^{T}$, the 
commutation relations can be rewritten as

$$
\left[R_{k}, R_{j}\right]=i \Omega_{k j},
$$

where $\Omega_{k j}$ are the elements of the symplectic matrix $\boldsymbol{\Omega}=$ $i \oplus_{k=1}^{n} \sigma_{2}, \sigma_{2}$ being the $y$-Pauli matrix. The covariance matrix $\sigma \equiv \sigma[\varrho]$ and the vector of mean values $\boldsymbol{X} \equiv \boldsymbol{X}[\varrho]$ of a quantum state $\varrho$ are defined as

$$
\begin{gathered}
X_{j}=\left\langle R_{j}\right\rangle, \\
\sigma_{k j}=\frac{1}{2}\left\langle\left\{R_{k}, R_{j}\right\}\right\rangle-\left\langle R_{j}\right\rangle\left\langle R_{k}\right\rangle,
\end{gathered}
$$

where $\{A, B\}=A B+B A$ denotes the anticommutator and $\langle O\rangle=\operatorname{Tr}[\varrho O]$ is the expectation value of the operator $O$.

A quantum state $\varrho_{G}$ is referred to as a Gaussian state if its characteristic function or, equivalently, the Wigner function have a Gaussian form, in the Cartesian notation

$$
\begin{gathered}
\chi\left[\varrho_{G}\right](\boldsymbol{\Lambda})=\exp \left\{-\frac{1}{2} \boldsymbol{\Lambda}^{T} \boldsymbol{\sigma} \boldsymbol{\Lambda}+\boldsymbol{X}^{T} \boldsymbol{\Omega} \boldsymbol{\Lambda}\right\}, \\
W\left[\varrho_{G}\right](\boldsymbol{Y})=\frac{\exp \left\{-\frac{1}{2}(\boldsymbol{Y}-\boldsymbol{X})^{T} \boldsymbol{\sigma}^{-1}(\boldsymbol{Y}-\boldsymbol{X})\right\}}{(2 \pi)^{n} \sqrt{\operatorname{Det}[\boldsymbol{\sigma}]}},
\end{gathered}
$$

where $\boldsymbol{\Lambda}$ and $\boldsymbol{Y}$ are real vectors,

$$
\begin{aligned}
& \boldsymbol{\Lambda}=\left(\operatorname{Re} \lambda_{1}, \operatorname{Im} \lambda_{1}, \ldots, \operatorname{Re} \lambda_{n}, \operatorname{Im} \lambda_{n}\right)^{T}, \\
& \boldsymbol{Y}=\left(\operatorname{Re} \alpha_{1}, \operatorname{Im} \alpha_{1}, \ldots, \operatorname{Re} \alpha_{n}, \operatorname{Im} \alpha_{n}\right)^{T} .
\end{aligned}
$$

Of course, once the covariance matrix and the vector of mean values are given, a Gaussian state is fully determined. For example, the purity $\mu\left[\varrho_{G}\right]=\operatorname{Tr}\left[\varrho_{G}^{2}\right]$ of an $n$-mode Gaussian state may be expressed as

$$
\mu\left[\varrho_{G}\right]=\frac{1}{2^{n} \sqrt{\operatorname{det} \sigma}} .
$$

An $n$-mode Gaussian state can be always written as

$$
\varrho_{G}=U_{S} \bigotimes_{k=1}^{n} v_{k}\left(n_{k}\right) U_{S}^{\dagger},
$$

where $v_{k}\left(n_{k}\right)=\left(1+n_{k}\right)^{-1}\left[n_{k} /\left(1+n_{k}\right)\right]^{a_{k}^{\dagger} a_{k}}$ is a single-mode thermal state with $n_{k}=\operatorname{Tr}\left[a_{k}^{\dagger} a_{k} v_{k}\left(n_{k}\right)\right]$ average number of photons and $U_{S}$ denotes the unitary evolution generated by a generic Hamiltonian at most bilinear in the mode operators, which is an evolution corresponding to a symplectic transformation in the phase space [96]. Any mapping, either unitary or completely positive, transforming Gaussian states into Gaussian states is a Gaussian operation.

For a single-mode system the most general Gaussian state can be written as

$$
\varrho_{G}=D(\alpha) S(\zeta) v(n) S^{\dagger}(\zeta) D^{\dagger}(\alpha),
$$

with $D(\alpha)$ being the displacement operator and $S(\zeta)=$ $\exp \left[\frac{1}{2} \zeta\left(a^{\dagger}\right)^{2}-\frac{1}{2} \zeta^{*} a^{2}\right]$ the single-mode squeezing operator with $\alpha, \zeta \equiv r e^{i \varphi} \in \mathbb{C}$. The corresponding covariance matrix has entries

$$
\begin{gathered}
\sigma_{11}=\left(n+\frac{1}{2}\right)[\cosh (2 r)-\sinh (2 r) \cos (\varphi)], \\
\sigma_{22}=\left(n+\frac{1}{2}\right)[\cosh (2 r)+\sinh (2 r) \cos (\varphi)], \\
\sigma_{12}=\sigma_{21}=\left(n+\frac{1}{2}\right) \sinh (2 r) \sin (\varphi)
\end{gathered}
$$

The Von-Neumann entropy $S(\varrho)=-\operatorname{Tr}[\varrho \ln \varrho]$ of a singlemode Gaussian state may be written as

$$
\begin{aligned}
\mathrm{S}\left(\varrho_{G}\right) & =h(\sqrt{\operatorname{det} \sigma})=h\left(\frac{1}{2 \mu}\right)=h\left(n+\frac{1}{2}\right) \\
& =(n+1) \ln (n+1)-n \ln (n),
\end{aligned}
$$

where we have introduced the function

$$
h(x)=\left(x+\frac{1}{2}\right) \ln \left(x+\frac{1}{2}\right)-\left(x-\frac{1}{2}\right) \ln \left(x-\frac{1}{2}\right) .
$$

For a two-mode Gaussian state, the covariance matrix (CM) is a real $4 \times 4$ symmetric-definite positive block matrix with ten independent parameters:

$$
\sigma=\left(\begin{array}{c|c}
A & C \\
\hline C^{T} & B
\end{array}\right) .
$$

Matrices $A, B$, and $C$ are $2 \times 2$ real matrices, representing, respectively, the autocorrelation matrices of the two modes and their mutual correlation matrix. Any two-mode CM $\sigma$ may be brought to its standard-form local symplectic operations, that is, local Gaussian operations. In the standard from, matrices $A$ and $B$ are proportional to the identity and $C$ is diagonal. Using the four local symplectic invariants $I_{1} \equiv \operatorname{det}(A), I_{2} \equiv \operatorname{det}(B)$, $I_{3} \equiv \operatorname{det}(C)$, and $I_{4} \equiv \operatorname{det}(\sigma)$, the symplectic eigenvalues, denoted by $d_{ \pm}$with $d_{-} \leqslant d_{+}$, read as follows:

$$
d_{ \pm}=\sqrt{\frac{\Delta(\sigma) \pm \sqrt{\Delta(\sigma)^{2}-4 I_{4}}}{2}}
$$

where $\Delta(\sigma) \equiv I_{1}+I_{2}+2 I_{3}$. Using the symplectic eigenvalues, the uncertainty relation can be rewritten as $d_{-} \geqslant 1 / 2$ and the Von-Neumann entropy as [97]

$$
\mathrm{S}\left(\varrho_{G}\right)=h\left(d_{-}\right)+h\left(d_{+}\right) .
$$

\section{QUANTUM NON-GAUSSIANITY MEASURES: DEFINITIONS AND PROPERTIES}

In this section we review the definitions of the $\mathrm{nG}$ measures for quantum states proposed in $[76,77]$ and illustrate in detail their properties. Although the two measures are based on different quantities, the Hilbert-Schmidt distance and the quantum relative entropy, they share the same basic idea: One wants to quantify the nG of a quantum state $\varrho$ in terms of the distinguishability of the state itself from a reference Gaussian state $\tau$, chosen as the Gaussian state with the same first and second moments of $\varrho$, such that

$$
\begin{aligned}
& X[\tau]=X[\varrho], \\
& \sigma[\tau]=\sigma[\varrho] .
\end{aligned}
$$

Notice that a similar line of reasoning has been adopted in Refs. [98-100] to define a measure of nonclassicality via the Hilbert-Schmidt distance. Here, roughly speaking, the two $\mathrm{nG}$ measures provide the quantization of the classical approaches to assess $\mathrm{nG}$ based on moments and negentropy, respectively. In the following we review their properties and provide a critical comparison with another quantities proposed in literature [78]. 


\section{A. Measuring the non-Gaussianity using Hilbert-Schmidt distance from a Gaussian reference}

Given two quantum states $\varrho_{1}$ and $\varrho_{2}$, the Hilbert-Schmidt distance is defined as

$$
\begin{aligned}
D_{\mathrm{HS}}\left[\varrho_{1}, \varrho_{2}\right] & =\left(\frac{1}{2} \operatorname{Tr}\left[\left(\varrho_{1}-\varrho_{2}\right)^{2}\right]\right)^{1 / 2} \\
& =\left(\frac{\mu\left[\varrho_{1}\right]+\mu\left[\varrho_{2}\right]-2 \kappa\left[\varrho_{1}, \varrho_{2}\right]}{2}\right)^{1 / 2},
\end{aligned}
$$

where $\mu[\varrho]$ is the purity of $\varrho$ and $\kappa\left[\varrho_{1}, \varrho_{2}\right]=\operatorname{Tr}\left[\varrho_{1} \varrho_{2}\right]$ denotes the overlap between $\varrho_{1}$ and $\varrho_{2}$. We define the degree of $n G$ of the state $\varrho$ as the squared renormalized HS distance [76],

$$
\delta_{A}[\varrho]=\frac{D_{\mathrm{HS}}^{2}[\varrho, \tau]}{\mu[\varrho]},
$$

of the state $\varrho$ from the state $\tau$, which is a reference Gaussian state chosen as in Eq. (23). The relevant properties of $\delta_{A}[\varrho]$ are summarized by the following lemmas.

Lemma A1. $\delta_{A}[\varrho]=0$ if and only if (iff) $\varrho$ is a Gaussian state.

Proof. If $\delta_{A}[\varrho]=0$, then $\varrho=\tau$ and thus it is a Gaussian state. If $\varrho$ is a Gaussian state, then it is uniquely identified by its first and second moments and thus the reference Gaussian state $\tau$ is given by $\tau=\varrho$, which, in turn, leads to $D_{\mathrm{HS}}[\varrho, \tau]=0$ and thus to $\delta_{A}[\varrho]=0$.

Lemma A2. If $U$ is a unitary map corresponding to a symplectic transformation in the phase space, that is, if $U=\exp \{-i H\}$ with Hermitian $H$ and at most bilinear in the field operators, then $\delta_{A}\left[U \varrho U^{\dagger}\right]=\delta_{A}[\varrho]$.

Proof. Let us consider $\varrho^{\prime}=U \varrho U^{\dagger}$. Then the covariance matrix transforms as $\sigma\left[\varrho^{\prime}\right]=\Sigma \sigma[\varrho] \Sigma^{T}, \Sigma$ being the symplectic transformation associated with $U$. At the same time the vector of mean values simply translates to $\boldsymbol{X}^{\prime}=\boldsymbol{X}+\boldsymbol{X}_{0}$, where $\boldsymbol{X}_{0}$ is the displacement generated by $U$. Since any Gaussian state is fully characterized by its first and second moments, then the reference state must necessarily transform as $\tau^{\prime}=U \tau U^{\dagger}$, that is, with the same unitary transformation $U$. Since the Hilbert-Schmidt distance and the purity of a quantum state are invariant under unitary transformations the lemma is proved.

This property ensures that single-mode displacement and squeezing operations, as well as two-mode evolutions such as those induced by a beam splitter or a parametric amplifier, do not change the Gaussian character of a quantum state. The lemma also allows us to always consider state with zero mean values.

Lemma $A 3 . \delta_{A}[\varrho]$ is proportional to the squared $L^{2}\left(\mathbb{C}^{n}\right)$ distance between the characteristic functions (or alternatively the Wigner functions) of $\varrho$ and of the reference Gaussian state $\tau$. This can be written as

$$
\begin{gathered}
\delta_{A}[\varrho] \propto \int d^{2 n} \lambda\{\chi[\varrho](\lambda)-\chi[\tau](\lambda)\}^{2}, \\
\left.\delta_{A}[\varrho] \propto \int d^{2 n} \boldsymbol{\alpha}\{W[\varrho](\boldsymbol{\alpha})-W[\tau](\boldsymbol{\alpha})]\right\}^{2} .
\end{gathered}
$$

Proof: Using the identities

$$
\operatorname{Tr}\left[O_{1} O_{2}\right]=\int \frac{d^{2 n} \lambda}{\pi^{n}} \chi\left[O_{1}\right](\lambda) \chi\left[O_{2}\right](-\lambda)
$$

$$
=\pi^{n} \int d^{2 n} \boldsymbol{\alpha} W\left[O_{1}\right](\boldsymbol{\alpha}) W\left[O_{2}\right](\boldsymbol{\alpha})
$$

and the fact the characteristic functions of self-adjoint operators are even functions of $\lambda$, we obtain

$$
\begin{aligned}
D_{\mathrm{HS}}^{2}[\varrho, \tau] & =\frac{1}{2} \int \frac{d^{2 n} \lambda}{\pi^{n}}\{\chi[\varrho](\boldsymbol{\lambda})-\chi[\tau](\boldsymbol{\lambda})\}^{2} \\
& =\frac{\pi^{n}}{2} \int d^{2 n} \lambda\{W[\varrho](\boldsymbol{\alpha})-W[\tau](\boldsymbol{\alpha})\}^{2},
\end{aligned}
$$

which proves the lemma.

Since the notion of Gaussianity of a quantum state is connected to the shape of its characteristic (Wigner) function, and since the characteristic function of a quantum state belongs to the $L^{2}\left(\mathbb{C}^{n}\right)$ space [95], we address $L^{2}(\mathbb{C})$ distance as a good indicator for the non-Gaussian character of $\varrho$.

Lemma A4. Consider a bipartite state $\varrho=\varrho_{A} \otimes \varrho_{G}$. If $\varrho_{G}$ is a Gaussian state, then $\delta_{A}[\varrho]=\delta_{A}\left[\varrho_{A}\right]$.

Proof. We have

$$
\begin{gathered}
\mu[\varrho]=\mu\left[\varrho_{A}\right] \mu\left[\varrho_{G}\right], \\
\mu[\tau]=\mu\left[\tau_{A}\right] \mu\left[\tau_{G}\right], \\
\kappa[\varrho, \tau]=\kappa\left[\varrho_{A}, \tau_{A}\right] \kappa\left[\varrho_{G}, \varrho_{G}\right] .
\end{gathered}
$$

Therefore, since $\kappa\left[\varrho_{G}, \varrho_{G}\right]=\mu\left[\varrho_{G}\right]$, we arrive at

$$
\begin{aligned}
\delta_{A}[\varrho] & =\frac{\mu\left[\varrho_{A}\right] \mu\left[\varrho_{G}\right]+\mu\left[\tau_{A}\right] \mu\left[\varrho_{G}\right]-2 \kappa\left[\varrho_{A}, \tau_{A}\right] \kappa\left[\varrho_{G}, \varrho_{G}\right]}{2 \mu\left[\varrho_{A}\right] \mu\left[\varrho_{G}\right]} \\
& =\delta_{A}\left[\varrho_{A}\right] .
\end{aligned}
$$

Notice, however, that $\delta_{A}[\varrho]$ is not generally additive (nor multiplicative) with respect to the tensor product. If we consider a (separable) multipartite quantum state in the product form $\varrho=\otimes_{k=1}^{n} \varrho_{k}$, the $\mathrm{nG}$ is given by

$$
\delta_{A}[\varrho]=\frac{\prod_{k=1}^{n} \mu\left[\varrho_{k}\right]+\prod_{k=1}^{n} \mu\left[\tau_{k}\right]-2 \prod_{k=1}^{n} \kappa\left[\varrho_{k}, \tau_{k}\right]}{2 \prod_{k=1}^{n} \mu\left[\varrho_{k}\right]},
$$

where $\tau_{k}$ is the Gaussian state with the same moments of $\varrho_{k}$. In fact, since the state $\varrho$ is factorizable, we have that the corresponding Gaussian $\tau$ is a factorizable state too.

For single-mode quantum states we have collected several numerical evidences that $\delta_{A}[\varrho]=1 / 2$ represents an upper bound for the HS nG of any quantum state [101]. The same conclusion is indirectly suggested by the results obtained in [83] and this leads to formulate the following conjecture.

Conjecture A1. For single-mode quantum states we have that $\delta_{A}[\varrho] \leqslant \frac{1}{2}$.

In particular, the conjecture has been numerically verified for single-mode CV states expressed as finite superposition of Fock number states, that is, for truncated states of the form $\varrho=\sum_{n, k=0}^{N} \varrho_{n k}|n\rangle\langle k|$. We have generated at random a large number states for various values of the truncating dimension $N$ and evaluated the corresponding $\mathrm{nG} \delta_{A}$. Results have shown that the value of the $\mathrm{nG} \delta_{A}$ is bounded by $1 / 2$ and the typical $\mathrm{nG}$ (the value of $\delta_{A}$ with the largest occurrence) decreases with both the purity and the truncating dimension. 


\section{B. Measuring the non-Gaussianity using the quantum relative entropy to a reference Gaussian}

Given two quantum states $\varrho_{1}$ and $\varrho_{2}$, the quantum relative entropy (QRE) is defined as

$$
\mathrm{S}\left(\varrho_{1} \| \varrho_{2}\right)=\operatorname{Tr}\left[\varrho_{1}\left(\ln \varrho_{1}-\ln \varrho_{2}\right)\right] .
$$

As for its classical counterpart, the Kullback-Leiber divergence, it can be demonstrated that $0 \leqslant S\left(\varrho_{1} \| \varrho_{2}\right)<\infty$ when it is definite, that is, when the support of the first state in the Hilbert space $\operatorname{supp} \varrho_{1} \subseteq \operatorname{supp} \varrho_{2}$, is contained in that of the second one. In particular, $S\left(\varrho_{1} \| \varrho_{2}\right)=0$ iff $\varrho_{1} \equiv \varrho_{2}$. This quantity, though not defining a proper metric in the Hilbert space (it is not symmetric in its arguments), has been widely used in different fields of quantum information as a measure of statistical distinguishability for quantum states [102,103] because of its nice properties and statistical meaning. In fact, if we consider two quantum states $\varrho$ and $\tau$ and perform $N$ measurements on $\varrho$, the probability of confusing $\varrho$ with $\tau$ is (for large $N) P_{N}(\varrho \rightarrow \tau) \sim \exp \{-N \mathrm{~S}(\varrho \| \tau)\}$.

The degree of $\mathrm{nG}$ of a state $\varrho$ may be quantified as [77]

$$
\delta_{B}[\varrho]=\mathrm{S}(\varrho \| \tau),
$$

where $\tau$ is the reference Gaussian state with the same first and second moments as in Eq. (23). Notice that, because of the choice of $\tau, \operatorname{Tr}[\tau \ln \tau]=\operatorname{Tr}[\varrho \ln \tau]$ and thus

$$
\delta_{B}[\varrho]=\operatorname{Tr}[\varrho \ln \varrho]-\operatorname{Tr}[\varrho \ln \tau]=\mathrm{S}(\tau)-\mathrm{S}(\varrho),
$$

where $S(\varrho)$ is the von Neumann entropy of a quantum state $\varrho$. The nG measure $\delta_{B}$ may be considered as the quantum analog of the negentropy introduced in Sec. II, where the differential entropy is replaced by the von Neumann entropy of the quantum states under investigation.

At fixed Von-Neumann entropy $\mathrm{nG}$ is determined by the first two moments of the canonical operators, which in turn uniquely determine the reference Gaussian state. Using formulas from Sec. III we may write explicit formulas of $\delta_{B}$ for single-mode and two-mode states:

$$
\begin{gathered}
\delta_{B}[\varrho]=h(\sqrt{\operatorname{det} \sigma})-\mathrm{S}(\varrho) \quad \text { single-mode state, } \\
\delta_{B}[\varrho]=h\left(d_{-}\right)+h\left(d_{+}\right)-\mathrm{S}(\varrho) \quad \text { two-mode state, }
\end{gathered}
$$

where $d_{ \pm}$are the symplectic eigenvalues of the two-mode CM and $h(x)$ is given in Eq. (19).

The relevant properties of $\delta_{B}[\varrho]$ are summarized by the following lemmas. As a matter of fact, the QRE measure of $n G$ has all the relevant properties proved for $\delta_{A}$ and shows additional properties concerning the evolution under generic (not unitary) Gaussian maps and under a tensor product.

Lemma B1. $\delta_{B}[\varrho]=0$ iff $\varrho$ is a Gaussian state.

Proof. If $\delta_{B}[\varrho]=0$, then $\varrho=\tau$ and thus it is a Gaussian state. If $\varrho$ is a Gaussian state, then it is uniquely identified by its first and second moments and thus the reference Gaussian state $\tau$ is given by $\tau=\varrho$, which, in turn, leads to $S(\varrho \| \tau)=0$ and thus to $\delta_{B}[\varrho]=0$.

Lemma B2. If $U$ is a unitary map corresponding to a symplectic transformation in the phase space, that is, if $U=\exp \{-i H\}$ with Hermitian $H$ that is at most bilinear in the field operators, then $\delta_{B}\left[U \varrho U^{\dagger}\right]=\delta_{B}[\varrho]$.
This property ensures that single-mode displacement and squeezing operations, as well as two-mode evolutions as those induced by a beam splitter or a parametric amplifier, do not change the Gaussian character of a quantum state. The lemma also allows us to always consider a state with zero mean values.

Proof. The lemma follows from the invariance of QRE under unitary operation.

Lemma B3. $\delta_{B}$ is additive for factorized states: $\delta_{B}\left[\varrho_{1} \otimes\right.$ $\left.\varrho_{2}\right]=\delta_{B}\left[\varrho_{1}\right]+\delta_{B}\left[\varrho_{2}\right]$. As a corollary we have that if $\varrho_{2}$ is a Gaussian state, then $\delta_{B}[\varrho]=\delta_{B}\left[\varrho_{1}\right]$.

Proof. The overall reference Gaussian state is the tensor product of the relative reference Gaussian states of $\varrho_{1}$ and $\varrho_{2}$, $\tau=\tau_{1} \otimes \tau_{2}$. The lemma follows from the additivity of QRE and the corollary from Lemma B1.

Lemma B4. $\delta_{B}$ monotonically decreases under partial trace; that is, given a bipartite state $\varrho$, then $\delta_{B}\left[\varrho_{A}\right] \leqslant \delta_{B}[\varrho]$ and $\delta_{B}\left[\varrho_{B}\right] \leqslant \delta_{B}[\varrho]$, where $\varrho_{A}=\operatorname{Tr}_{B}[\varrho], \varrho_{B}=\operatorname{Tr}_{A}[\varrho]$.

Proof. Let us consider the partial trace state $\varrho_{A}\left(\varrho_{B}\right)$. Its $\mathrm{CM}$ is the submatrix of $\sigma[\varrho]$ obtained by dropping lines and rows involving expectation values on the system $B(A)$. Analogously, the first moment vector is the proper subvector of $\boldsymbol{X}[\varrho]$. Therefore, the reference Gaussian state $\tau_{A}\left(\tau_{B}\right)$ must necessarily satisfy $\tau_{A}=\operatorname{Tr}_{B}[\tau]$, where $\tau$ is the Gaussian reference of $\varrho\left(\tau_{B}=\operatorname{Tr}_{A}[\tau]\right)$. The QRE monotonically decreases under partial trace and thus the lemma is proved.

Actually, the preceding statement can be strengthened, as expressed by the following lemma.

Lemma B5. Given a generic bipartite state $\varrho$, we have $\delta[\varrho] \geqslant$ $\delta\left[\varrho_{A}\right]+\delta\left[\varrho_{B}\right]$.

Proof. It has been shown in [104] that QRE decreases monotonically under a generic (nonlinear) coarse graining. A simple example of nonlinear coarse graining that cannot be obtained via a completely positive quantum map is the operation $\varrho \rightarrow \varrho_{A} \otimes \varrho_{B}$. Because of this property we have $\delta[\varrho] \geqslant \delta\left[\varrho_{A} \otimes \varrho_{B}\right]=\delta\left[\varrho_{A}\right]+\delta\left[\varrho_{B}\right]$, where in the last equality we have used Lemma B3.

As an example, let us consider the class of pure two-mode photon-number entangled states (PNES),

$$
\left.\left|\psi_{P}\right\rangle\right\rangle=\sum_{n} \psi_{n}|n\rangle|n\rangle,
$$

together with their (equal) partial traces $\varrho_{A}$ and $\varrho_{B}$, that is the diagonal mixtures of Fock states given by

$$
\varrho_{P}=\sum_{n}\left|\psi_{n}\right|^{2}|n\rangle\langle n| .
$$

Relevant examples of non-Gaussian PNES are given by the photon-subtracted squeezed vacua (PSSV) $\psi_{n} \propto(n+1) x^{n+1}$ and the photon-added two-mode squeezed vacua (PASV) $\psi_{n} \propto n x^{n-1}$, which are obtained from the Gaussian PNES $\psi_{n} \propto x^{n} 0 \leqslant x<1$ (twin-beam state) by the experimentally feasible operations of photon subtraction $\varrho \rightarrow a_{1} a_{2} \varrho a_{1}^{\dagger} a_{2}^{\dagger}$ and addition $\varrho \rightarrow a_{1}^{\dagger} a_{2}^{\dagger} \varrho a_{1} a_{2}$, respectively [36]. The pair-coherent or two-mode coherently correlated states (TMCs) [105-107] with Poissonian profile $\psi_{n} \propto \frac{\lambda^{n}}{n !}, \lambda \in \mathbb{R}$. The mean energy of PNES is $N_{P}=\left\langle\left\langle\psi\left|a_{1}^{\dagger} a_{1}+a_{2}^{\dagger} a_{2}\right| \psi\right\rangle\right\rangle \equiv 2 N$, where $N=$ $\sum_{n=0}^{\infty}\left|\psi_{n}\right|^{2} n$, whereas correlations between the modes can be quantified by $C=\operatorname{Re} \sum_{n=0}^{\infty} \psi_{n}^{*} \psi_{n+1}(n+1)$ and entanglement is given by the Von-Neumann entropy of the partial traces 


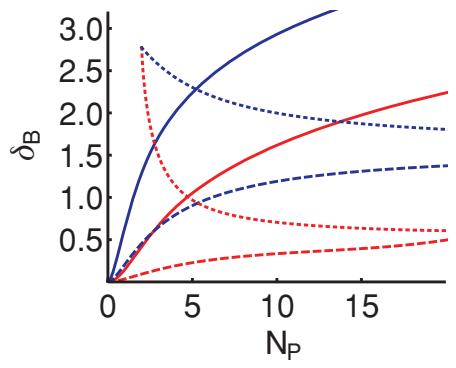

FIG. 2. (Color online) Non-Gaussianity $\delta_{B}\left[\psi_{P}\right]$ of PNES states (blue upper curves) and of their partial traces $2 \delta_{B}\left[\varrho_{P}\right]$ (red lower curves) as a function of the total energy of the PNES. Solid curves are for TMC, dashed for PSSV, and dotted for PASV.

$\epsilon_{0}=-\sum_{n} \psi_{n}^{2} \ln \psi_{n}^{2}$. In turn, the covariance matrix of a PNES equals that of a symmetric Gaussian state in standard form, with diagonal elements equal to $N+\frac{1}{2}$ and off-diagonal blocks given by $\boldsymbol{C}=\operatorname{diag}(C,-C)$. In Fig. 2 we report the $\mathrm{nG}$ $\delta_{B}\left[\psi_{P}\right]$ of PNESs as a function of the overall energy $N_{P}$ together with the sum of the $\mathrm{nG}$ of the partial traces, that is, $\delta_{B}\left[\varrho_{A}\right]+\delta_{B}\left[\varrho_{b}\right]=2 \delta_{B}\left[\varrho_{P}\right]$. As predicted by the previous lemmas, the $\mathrm{nG}$ of a PNES state is always larger than the one of its partial traces and also of their sum.

Lemma B6. $\delta_{B}[\varrho]$ monotonically decreases under Gaussian quantum channels; that is $\delta\left[\mathcal{E}_{G}(\varrho)\right] \leqslant \delta[\varrho]$.

Proof. Any Gaussian quantum channel can be written as $\mathcal{E}_{G}(\varrho)=\operatorname{Tr}_{E}\left[U_{b}\left(\varrho \otimes \tau_{E}\right) U_{b}^{\dagger}\right]$, where $U_{b}$ is a unitary operation corresponding to a Hamiltonian at most bilinear in the field modes and where $\tau_{E}$ is a Gaussian state [108]. Then, by using Lemmas B2, B3, and B4, we obtain $\delta_{B}\left[\mathcal{E}_{G}(\varrho)\right] \leqslant \delta\left[U_{b}(\varrho \otimes\right.$ $\left.\left.\tau_{E}\right) U_{b}^{\dagger}\right]=\delta_{B}[\varrho]$.

In turn, this lemma provides a necessary condition for a channel to be Gaussian: Given a quantum channel $\mathcal{E}$ and a generic quantum state $\varrho$, if the inequality $\delta_{B}[\mathcal{E}(\varrho)] \leqslant \delta_{B}[\varrho]$ is not fulfilled, the channel is non-Gaussian. It is also worth noticing that the monotonicity is fulfilled only for a proper completely positive (CP) map. As we see in Sec. VII, even if we consider the conditional evolution corresponding to a Gaussian measurement operator, the $\mathrm{nG}$ of the output states may increase. Indeed, in this case, we do not consider a full CP map, but only one Krauss operator corresponding to the chosen (Gaussian) measurement operator.

Lemma B7. For a set of states $\left\{\varrho_{k}\right\}$ having the same first and second moments, then $\mathrm{nG}$ is a convex functional, that is,

$$
\delta_{B}\left[\sum_{k} p_{k} \varrho_{k}\right] \leqslant \sum_{k} p_{k} \delta_{B}\left[\varrho_{i}\right],
$$

with $\sum_{k} p_{k}=1$.

Proof. The states $\varrho_{k}$, having the same first and second moments, have the same reference Gaussian state $\tau$ which in turn is the reference Gaussian state of the convex combination $\varrho=$ $\sum_{k} p_{k} \varrho_{k}$. Since conditional entropy $\mathrm{S}(\varrho \| \tau)$ is a jointly convex functional with respect to both states, we have $\delta_{B}\left[\sum_{k} p_{k} \varrho_{k}\right]=$ $\mathrm{S}\left(\sum_{k} p_{k} \varrho_{k} \| \tau\right) \leqslant \sum_{k} p_{k} \mathrm{~S}\left(\varrho_{k} \| \tau\right)=\sum_{k} p_{k} \delta\left[\varrho_{k}\right]$.

Notice that, in general, $\delta_{B}$ is not convex, as it may easily proved upon considering the convex combination of two Gaussian states with different parameters.
Lemma B8. At a fixed average number of photons $N=$ $\left\langle a^{\dagger} a\right\rangle$, the maximum value of $\mathrm{nG}$ measured by $\delta_{B}$ for singlemode states is achieved by pure superpositions of Fock states $\left|\psi_{N}\right\rangle=\sum_{k} \alpha_{k}\left|n+l_{k}\right\rangle$, where $n \geqslant 0, l_{k} \geqslant l_{k-1}+3$ or $l_{k}=0$, and with the constraint $N=\{\operatorname{det} \sigma[v(N)]\}^{\frac{1}{2}}-\frac{1}{2}=$ $n+\sum_{k}\left|\alpha_{k}\right|^{2} l_{k}$.

Proof. Since $\delta_{B}[\varrho]=\mathrm{S}(\tau)-\mathrm{S}(\varrho)$ we have to maximize $\mathrm{S}(\tau)$ and, at the same time, minimize $\mathrm{S}(\varrho)$. For a single-mode system the most general Gaussian state can be written as $\varrho_{G}=$ $D(\alpha) S(\zeta) v\left(n_{t}\right) S^{\dagger}(\zeta) D^{\dagger}(\alpha)$, with $D(\alpha)$ being the displacement operator, $S(\zeta)$ the squeezing operator, $\alpha, \zeta \in \mathbb{C}$, and $v\left(n_{t}\right)$ a thermal state with $n_{t}$ average number of photons. Displacement and squeezing applied to thermal states increase the overall energy, while entropy is an increasing monotonous function of the number of thermal photons $n_{t}$ and is invariant under unitary operations. Thus, at fixed energy, $\mathrm{S}(\tau)$ is maximized for $\tau=v(N)$. Therefore, the state with the maximum amount of $\mathrm{nG}$ must be a pure state [in order to have $\mathrm{S}(\varrho)=0$ ] with the same CM $\sigma=\left(N+\frac{1}{2}\right) \mathbb{I}$ of the thermal state $v(N)$. One can easily check now that the state with this property is the one indicated in the lemma. One can also observe that by choosing $n=N$ and $l_{k}=0$, we obtain that Fock states $|N\rangle$ are maximum non-Gaussian states at fixed energy.

As is clear from the examples presented in the next sections, the two $\mathrm{nG}$ measures induce different ordering on the set of quantum states; that is, we may find a pair of states $\varrho_{1}$ and $\varrho_{2}$ such that $\delta_{A}\left[\varrho_{1}\right]>\delta_{A}\left[\varrho_{2}\right]$ and $\delta_{B}\left[\varrho_{1}\right]<\delta_{B}\left[\varrho_{2}\right]$, or vice versa. One may conjecture that, as it happens for entanglement measures [109], we indeed do not have a unique $\mathrm{nG}$ measure and that different measures correspond to different operational meanings. As also remarked in Sec. VIII, an operational meaning for $\delta_{B}$ may be found in terms of information-theoretic quantities, while an operational meaning for $\delta_{A}$, besides its connection with the distance in the phase space, is still missing. The two measures are connected to each other by means of the inequality $S(\varrho \| \tau) \geqslant D_{\mathrm{HS}}^{2}[\varrho, \tau][110]$, which, in turn, implies the inequality

$$
\delta_{B}[\varrho] \geqslant \delta_{A}[\varrho] \mu[\varrho] .
$$

For pure states, (39) reduces to $\delta_{B}[\varrho] \geqslant \delta_{A}[\varrho]$.

\section{A measure of non-Gaussianity based on the Wehrl entropy}

A different measure of $\mathrm{nG}$ has been proposed in [78], based on the difference between the Wehrl entropies of the reference Gaussian state and the quantum state in exam,

$$
\delta_{C}[\varrho]=H_{W}(\tau)-H_{W}(\varrho)
$$

where

$$
H_{W}(\varrho)=-\int_{\mathbb{C}} d^{2} \alpha Q_{\varrho}(\alpha) \ln \left[\pi Q_{\varrho}(\alpha)\right]
$$

is the Wehrl entropy, that is, the differential entropy of the normalized $Q$-Husimi function,

$$
Q_{\varrho}(\alpha)=\frac{1}{\pi}\left\langle 0\left|D(\alpha)^{\dagger} \varrho D(\alpha)\right| 0\right\rangle,
$$

of the state $\varrho$. The quantity $\delta_{C}$ owns reasonable properties in the phase space, which are inherited from those of the the $Q$ function. However, it lacks an operational meaning and 


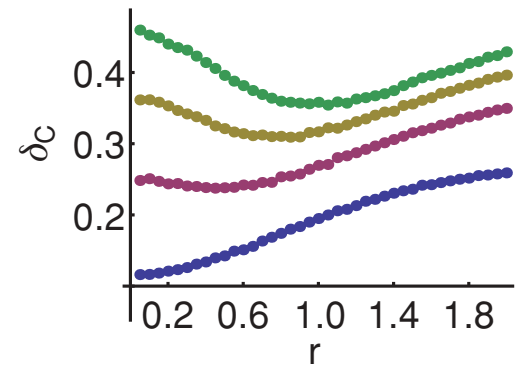

FIG. 3. (Color online) The Wehrl entropy-based $\mathrm{nG}$ $\delta_{C}\left[S(r)|n\rangle\langle n| S(r)^{\dagger}\right]$ for squeezed Fock states as a function of the squeezing parameter $r$. From bottom to top, the $\mathrm{nG}$ for $n=1,2,3,4$.

is not invariant under Gaussian unitary operations. In order to illustrate this behavior we have (numerically) evaluated the $\mathrm{nG}$ $\delta_{C}$ for Fock number states $|n\rangle$ subjected to squeezing. In Fig. 3 we show $\delta_{C}\left[S(r)|n\rangle\langle n| S(r)^{\dagger}\right]$ as a function of $r$ for different values of $n$. As is apparent from the plot, the $\mathrm{nG}$ is neither constant nor monotone with the squeezing parameter $r$.

\section{Non-Gaussianity of a quantum operation}

Once one obtains a good measure for the $\mathrm{nG}$ of quantum state, this may be exploited to define a measure for the nonGaussian character of a quantum operation. Let us denote by $\mathcal{G}$ the whole set of Gaussian states. A convenient definition for the $\mathrm{nG}$ of a map $\mathcal{E}$ reads as follows:

$$
\delta[\mathcal{E}]=\max _{\varrho \in \mathcal{G}} \delta[\mathcal{E}(\varrho)],
$$

where $\mathcal{E}(\varrho)$ denotes the quantum state obtained after the evolution imposed by the map. Indeed, we have $\delta\left[\mathcal{E}_{g}\right]=0$ iff $\mathcal{E}$ is a Gaussian map $\mathcal{E}_{g}$, that is, a map which transforms any input Gaussian state into a Gaussian state. Other properties follow from those of the $\mathrm{nG}$ measures for states.

Despite the conceptual simplicity of the preceding definition, the evaluation of $\delta[\mathcal{E}]$ is, in general, a challenging task using either the HS-based or the QRE-based measure of $\mathrm{nG}$. As a consequence, it has not been used so far for a systematic classification of maps.

\section{NON-GAUSSIANITIES OF SPECIFIC FAMILIES OF QUANTUM STATES}

This section is devoted to a sort of zoology of $\mathrm{nG}$; that is, we consider different families of relevant quantum states and evaluate their non-Gaussianities, $\delta_{A}$ and $\delta_{B}$. In this way, we analyze in some detail the relationships between the two measures and illustrate their basic features also in connection with the analytical properties of their density operators and the intuition coming from their phase-space quasidistributions.

\section{A. Fock states and superpositions}

We consider single-mode Fock states $|n\rangle$ and superpositions of Fock states of the form

$$
\left|\psi_{n k}\right\rangle=\frac{1}{\sqrt{2}}[|n\rangle+|n+k\rangle]
$$

for $n>0$ and $k>2$. The reference Gaussian states are thermal states $\tau_{n k}=v\left(n+\frac{k}{2}\right)$ with $n+\frac{k}{2}$ average photons. NonGaussianity can be analytically evaluated for both measures, obtaining (for $k=0$ and $k>2$ )

$$
\begin{gathered}
\delta_{A}\left[\psi_{n k}\right]=\frac{1}{2}\left(1+\frac{1}{2 n+k}-2 O_{n k}\right), \\
\delta_{B}\left[\psi_{n k}\right]=h\left(n+\frac{k+1}{2}\right),
\end{gathered}
$$

where the overlap $O_{n k}=\left\langle\psi_{n k}\left|\nu\left(n+\frac{k}{2}\right)\right| \psi_{n k}\right\rangle$ is given by

$$
O_{n k}=\frac{1}{2}\left[\frac{\left(n+\frac{k}{2}\right)^{n}}{\left(n+\frac{k}{2}+1\right)^{1+n}}+\frac{\left(n+\frac{k}{2}\right)^{n+k}}{\left(n+\frac{k}{2}+1\right)^{1+n+k}}\right] .
$$

As is apparent from Fig. 4, both measures increase with both $n$ and $k$ and are monotone functions of each other for these families of states; that is, if $\delta_{A}\left[\varrho_{1}\right]>\delta_{A}\left[\varrho_{2}\right]$, then $\delta_{B}\left[\varrho_{1}\right]>$ $\delta_{B}\left[\varrho_{2}\right]$. As stated in Lemma B7 Fock states have the maximum $\mathrm{nG}$ at fixed number of photons according to the measure $\delta_{B}$. Though it has not been proved yet, we observe the same result for the HS-based $\delta_{A}$ in all the examples considered up to now.

\section{B. Mixtures of Fock states}

We now investigate the monotonicity of the two measures for other one-parameter families of quantum states. In this case we consider mixtures of Fock states of the form

$$
\varrho_{D}=\sum_{n=0}^{\infty} q_{n}(\lambda)|n\rangle\langle n|,
$$

where $0 \leqslant q_{n}(\lambda) \leqslant 1, \sum_{n} q_{n}(\lambda)=1$, and $\bar{n}_{\lambda}=\sum_{n} n q_{n}(\lambda)$ is the average photon number of the state. The reference Gaussian state for any diagonal mixtures of Fock states is a thermal state $v\left(\bar{n}_{\lambda}\right)$ with the same average photon number. The $\mathrm{nG}$ can be thus written as

$$
\begin{aligned}
& \delta_{A}\left[\varrho_{D}\right]=\frac{1}{2}\left[1-\frac{\sum_{n} \tau_{n}\left(2 q_{n}-\tau_{n}\right)}{\sum_{n} q_{n}^{2}}\right], \\
& \delta_{B}\left[\varrho_{D}\right]=h\left(\bar{n}_{\lambda}+1 / 2\right)+\sum_{n} q_{n} \ln q_{n},
\end{aligned}
$$

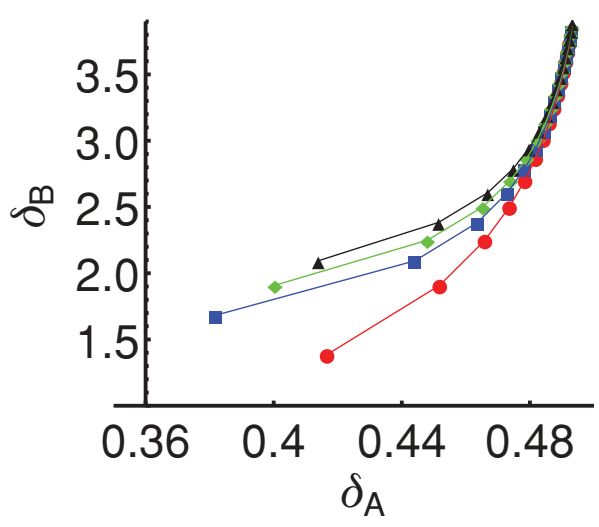

FIG. 4. (Color online) QRE-based $\mathrm{nG} \delta_{B}$ as a function of HS distance $\mathrm{nG} \delta_{A}$ for Fock states $|n\rangle$ with $n=1, \ldots, 15$ (red circles) and for superpositions $\left|\psi_{n k}\right\rangle$ with $n=1, \ldots 15$ and $k=3$ (blue squares), $k=4$ (green diamonds), and $k=5$ (black triangles). 
where $\tau_{n}=\left\langle n\left|v\left(\bar{n}_{\lambda}\right)\right| n\right\rangle$ are the matrix elements of the (thermal) Gaussian reference state. We have numerically evaluated the non-Gaussianities for several one-parameter families including the diagonal states obtained as partial traces of TMS, PSSV, and PASV states [see Eq. (38) and the discussion following Lemma B4], as well as diagonal states with Poissonian profile or given by a $\Gamma$ distribution of the form $q_{n}^{(k)}(\lambda) \propto n^{k} \exp \{-n / \lambda\}$. Results are shown in the left panel of Fig. 5: The two measures are monotone to each other for all the considered families and the behavior of $\delta_{B}\left[\varrho_{D}\right]$ vs $\delta_{A}\left[\varrho_{D}\right]$ is almost independent of the kind of states. These results suggest that a general relation between the two measures may exist for mixtures of Fock states. However, so far we have not been able to prove it analytically starting from the expressions of $\delta_{A}\left[\varrho_{D}\right]$ and $\delta_{B}\left[\varrho_{D}\right]$ in Eqs. (45).

We have also considered (truncated) random mixtures of the form

$$
\varrho_{H}=\sum_{n=0}^{H} p_{n}|n\rangle\langle n|,
$$

where $H$ is the truncation dimension, and have numerically evaluated the non-Gaussianities using Eqs. (45) for a sample of $10^{4}$ states. Results are reported in the right panel of Fig. 5 and show that despite the large number of involved parameters (up to $H=1000$ ) the two measures are almost monotone to each other. As $H$ increases, the distribution of the two measures concentrates around the typical values.

\section{Schrödinger cat states}

Let us now consider the two-parameter family of quantum states given by the Schrödinger cat-like states, that is, superpositions of coherent states $|\alpha\rangle=D(\alpha)|0\rangle$ and $|-\alpha\rangle$,

$$
\left|\psi_{S}\right\rangle=\frac{\cos \phi|\alpha\rangle+\sin \phi|-\alpha\rangle}{\sqrt{1+\sin (2 \phi) \exp \left\{-2 \alpha^{2}\right\}}} .
$$

For $\phi= \pm \pi / 4,\left|\psi_{S}\right\rangle$ reduces to the so-called odd and even Schrödinger cat states. Using the fact that the reference
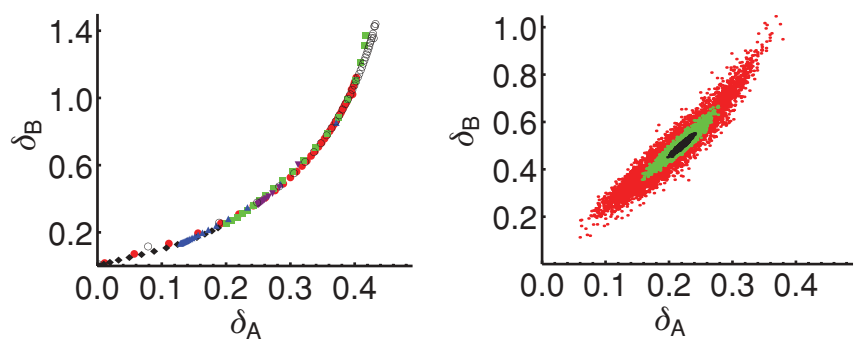

FIG. 5. (Color online) (Left) QRE-based $\mathrm{nG} \delta_{B}$ as a function of HS distance nG $\delta_{A}$ for mixtures of Fock states $\varrho_{D}$. Solid red circles refer to a Poissonian distribution, whereas open black circles are for the diagonal states obitained as partial trace of TMC; green squares and black diamonds are for mixtures coming from partial traces of photon-added and photon-subtracted two-mode squeezed vacuum, respectively; blue and purple triangles correspond to $\Gamma$ distributions $q_{n}^{(2)}(\lambda)$ and $q_{n}^{(4)}(\lambda)$, respectively. (Right) QRE-based $\mathrm{nG}$ $\delta_{B}$ as a function of HS distance $\mathrm{nG} \delta_{A}$ for a sample of $10^{4}$ truncated random mixtures $\varrho_{H}$ of Fock states. The red cloud refers to $H=10$, green for $H=100$, and black for $H=1000$.
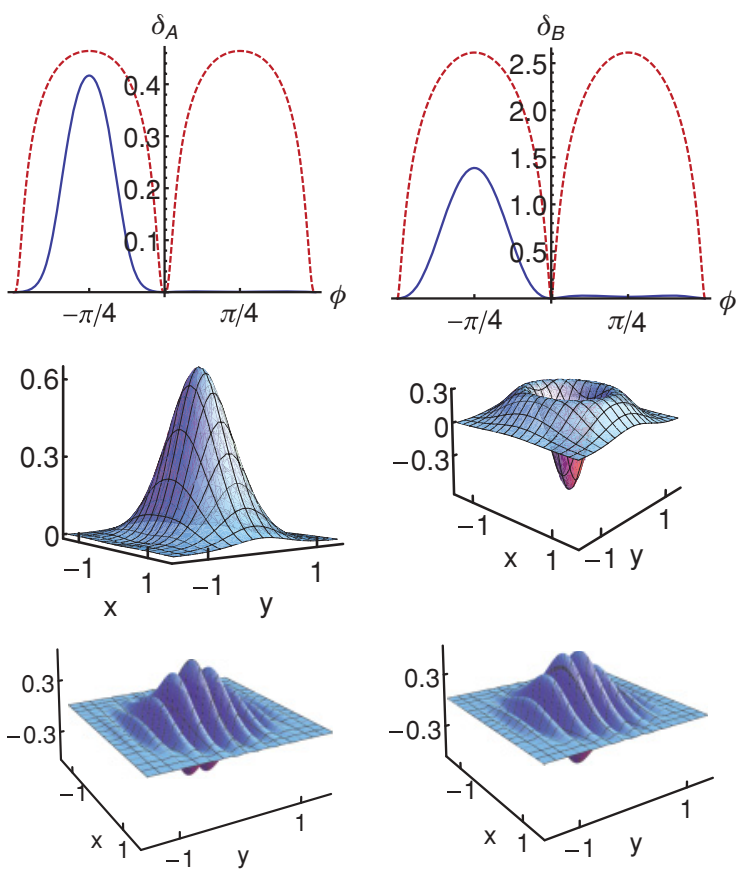

FIG. 6. (Color online) HS-based $\mathrm{nG} \delta_{A}$ (topmost left panel) and QRE-based $\mathrm{nG} \delta_{B}$ (topmost right panel) for Schrödinger cat states $\left|\psi_{S}\right\rangle$ as a function of $\phi$ and for different values of the amplitude $\alpha$. Solid bue line, $\alpha=0.5$; red dashed line, $\alpha=5$. The corresponding Wigner functions for the odd and the even cats are also shown in the lower panels: $\alpha=0.5, \phi=+\pi / 4$ (top left); $\alpha=0.5, \phi=-\pi / 4$ (top right); $\alpha=5, \phi=+\pi / 4$ (bottom left); $\alpha=5, \phi=-\pi / 4$ (bottom right).

Gaussian state is a displaced squeezed thermal state $\tau_{S}=$ $D(C) S(r) v(N) S^{\dagger}(r) D^{\dagger}(C)$, where the real parameters $C, r$, and $N$ are analytical function of $\phi$ and $\alpha$, we have evaluated the $\mathrm{nG}$ measures $\delta_{A}$ and $\delta_{B}$ for different values of $\alpha$ and $\phi$. Results are shown in Fig. 6.

As is apparent from the plots, upon varying the value of the parameters the two measures exhibit similar qualitative behavior. In particular, for low values of the amplitude (e.g., $\alpha=0.5$ ), we observe an asymmetric behavior with respect to $\phi: \mathrm{nG}$ is almost zero for positive $\phi$, while for $\phi<0$ one achieve high values of nG. By increasing the value of the amplitude, say $\alpha=5$, both measures become even functions of $\phi$. This can be understood by looking at the Wigner functions of even and odd Schrödinger cat states. In fact, for low amplitudes, the even cat $(\phi=\pi / 4)$ Wigner function is similar to a Gaussian state, in particular to the vacuum state, while for $\phi=-\pi / 4$ it presents a non-Gaussian hole in the origin of the phase space; for higher values of $\alpha$, one can observe similar non-Gaussian beahviors both for the even and the odd cat state.

Although the two nG measures capture the same qualitative non-Gaussian behavior, it is apparent from the parametric plot of Fig. 7 that they induce different ordering on the set of states. In fact, upon varying the two parameters $\alpha$ and $\phi$, one may find pair of states $\varrho_{1}, \varrho_{2}$ for which $\delta_{A}\left[\varrho_{1}\right]>\delta_{A}\left[\varrho_{2}\right]$ and $\delta_{B}\left[\varrho_{1}\right]<\delta_{B}\left[\varrho_{2}\right]$. As discussed before, we do accept that the two measures may induce different ordering on quantum states. Notice, however, that upon fixing one of the parameters and varying the other, one observes again a monotonous behavior: 


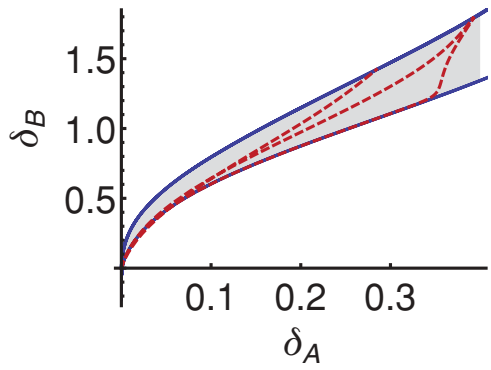

FIG. 7. (Color online) QRE-based $\mathrm{nG} \delta_{B}$ as a function of HS distance $\mathrm{nG} \delta_{A}$ for Schrödinger cat states $\left|\psi_{S}\right\rangle$. Solid blue lines refer to fixed amplitudes of coherent states $\alpha$, varying the angle $-\pi / 2<$ $\phi<\pi / 2$; from bottom to top, we have $\alpha=0.5,2.5$. The dashed red lines are for fixed angles $\phi$ and varying the amplitude $0<\alpha<2.5$; from bottom to top, $\phi=-\pi / 3, \pi / 6,2 \pi / 5$. The gray area denotes all the allowed values for the two $\mathrm{nG}$ measures for the considered range of values of the two parameters.

the red dashed lines in Fig. 7 show $\delta_{B}$ vs $\delta_{A}$ for fixed values of $\phi$ and varying the amplitude $\alpha$. This appears to be a typical behavior: For all the one-parameter families of states we have considered so far, the two measures are monotone with respect to each other and induce the same ordering of $\mathrm{nG}$.

\section{GAUSSIFICATION AND DE-GAUSSIFICATION PROCESSES}

In this section we consider a single-mode Gaussification, the loss mechanism due to the interaction with a bath of harmonic oscillators at zero temperature, and two de-Gaussification processes due either to phase diffusion or Kerr interaction. Other Gaussification and de-Gaussification protocols are considered in the next section, where we discuss the case of entanglement distillation.

\section{A. Loss mechanism}

The evolution of a single-mode quantum state interacting with a bath of harmonic oscillators at zero temperature is described by the Lindblad master equation

$$
\dot{\varrho}=\frac{\gamma}{2} \mathcal{L}[a] \varrho,
$$

where $\dot{\varrho}$ denotes time derivative, $\gamma$ is the damping factor, and the superoperator $\mathcal{L}[O]$ acts as follows:

$$
\mathcal{L}[O] \varrho=2 O^{\dagger} \varrho O-O^{\dagger} O \varrho-\varrho O^{\dagger} O
$$

Upon writing $\eta=e^{-\gamma t}$ the solution of the master equation can be written as

$$
\begin{gathered}
\varrho(\eta)=\sum_{m} V_{m} \varrho V_{m}^{\dagger}, \\
V_{m}=\sqrt{\frac{(1-\eta)^{m}}{m !}} a^{m} \eta^{\frac{1}{2}\left(a^{\dagger} a-m\right)},
\end{gathered}
$$

where $\varrho$ is the initial state. In particular, if the system is initially prepared in a Fock state $\varrho_{p}=|p\rangle\langle p|$, after the evolution we obtain the mixed state

$$
\varrho_{p}(\eta)=\sum_{m} V_{m} \varrho_{p} V_{m}^{\dagger}=\sum_{l=0}^{p} \alpha_{l, p}(\eta)|l\rangle\langle l|,
$$

with

$$
\alpha_{l, p}(\eta)=\left(\begin{array}{l}
p \\
l
\end{array}\right)(1-\eta)^{p-l} \eta^{l} .
$$

Since the state is diagonal in the Fock basis, the reference Gaussian state is a thermal state $\tau_{p}(\eta)=v(p \eta)$ with average photon number $p \eta$. Non-Gaussianity $\delta_{A}$ can be evaluated analytically,

$$
\begin{aligned}
\delta_{A}= & \frac{1}{2(1-\eta)^{2 m}{ }_{2} F_{1}\left(-m,-m, 1 ; \frac{\eta^{2}}{(\eta-1)^{2}}\right)} \\
& \times\left\{(1-\eta)^{2 m}{ }_{2} F_{1}\left(-m,-m, 1 ; \frac{\eta^{2}}{(\eta-1)^{2}}\right)\right. \\
& \left.+(1+2 m \eta)^{-1}-\frac{2[1+(m-1) \eta]^{m}}{(1+m \eta)^{m+1}}\right\},
\end{aligned}
$$

where ${ }_{2} F_{1}(a, b, c, ; x)$ denotes the hypergeometric function, and $\delta_{B}$ can be evaluated numerically via the formula

$$
\begin{aligned}
\delta_{B}= & p \eta \ln \left(\frac{p \eta+1}{p \eta}\right)+\ln (1+p \eta) \\
& +\sum_{l=0}^{\infty} \alpha_{l, p}(\eta) \ln \left[\alpha_{l, p}(\eta)\right] .
\end{aligned}
$$

Because of Lemma B6 we know for sure that $\delta_{B}$ is decreasing with time $\eta t$, while this property is not guaranteed for $\delta_{A}$. In Fig. 8 we plot both non-Gaussianities as a function of dimensionless time $\eta t$ and for different values of $p$, observing that also $\delta_{A}$ is decreasing with time and that both are monotonically increasing functions of $p$, that is, at fixed time $t$ the higher the initial photon number $p$, the larger is the $\mathrm{nG}$ of the evolved state. Although they present a different shape, due also to their different scale ( $\delta_{A}$ for single-mode states is bounded by $1 / 2$, while $\delta_{B}$ is in general unbounded), we observe a similar trend for both $\mathrm{nG}$ which, in particular, approach zero for the same values of the parameters $\eta, t$, and $p$.
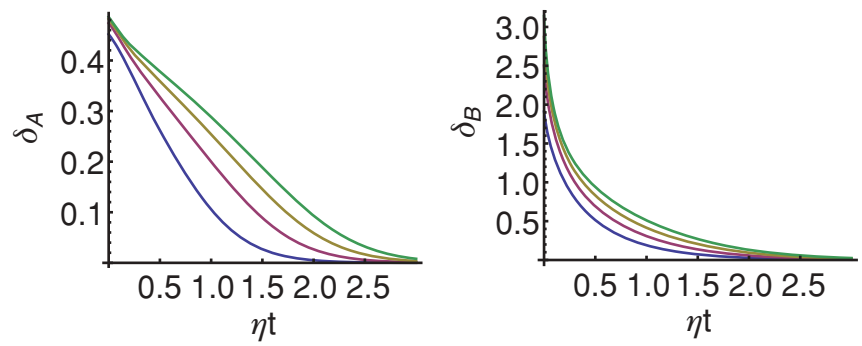

FIG. 8. (Color online) HS-based $\mathrm{nG} \delta_{A}$ (left) and QRE-based nG $\delta_{B}$ (right) for a Fock state $|p\rangle$ under loss mechanism as a function of dimensionless time $\eta t$ and for different values of $p$. From bottom to top, $p=\{2,4,6,8\}$. 


\section{B. Phase-diffusion evolution}

Let us consider single-mode systems evolving according to the following master equation:

$$
\dot{\varrho}=\Gamma \mathcal{L}\left[a^{\dagger} a\right] \varrho,
$$

where the superoperator $\mathcal{L}[A]$ has been defined in Eq. (48) and $\Gamma$ is the noise factor. This master equation describes the evolution of a quantum state subjected to a phase-diffusive noise. These non-Gaussian fluctuations are an important source of noise in optical communication links, and protocols able to purify squeezing or distilling entanglement have been recently proposed $[46,48]$. Upon writing $\varrho$ in the Fock state basis, the master equation leads to differential equations for the matrix elements $\varrho_{n m}=\langle n|\varrho| m\rangle$, where $\dot{\varrho}_{n m}=-\frac{1}{2} \Gamma(n-$ $m)^{2} \varrho_{n m}$, whose solutions read as follows:

$$
\varrho_{n m}(t)=e^{-\Delta^{2}(n-m)^{2}} \varrho_{n m}(0) .
$$

In the preceding equation we defined $\Delta^{2} \equiv \Gamma t / 2$, whereas $\varrho_{n m}(0)$ denote the matrix elements of the initial state. From Eq. (53) it is clear that the off-diagonal elements of the density matrix are progressively destroyed, whereas the diagonal ones are left unchanged and, in turn, energy is conserved.

It is worth noting that the same evolution as in (53) can be also obtained by the application of a random, zero-mean Gaussian-distributed phase-shift to the quantum state. Since the phase shift of an amount $\varphi$ is described by the unitary operator $U_{\varphi} \equiv \exp \left(-i \varphi a^{\dagger} a\right)$, we can write the state degraded by the Gaussian phase noise as follows:

$$
\begin{aligned}
\varrho_{\mathrm{Gn}} & =\int_{\mathbb{R}} d \varphi \frac{e^{-\varphi^{2} /\left(4 \Delta^{2}\right)}}{\sqrt{4 \pi \Delta^{2}}} U_{\varphi} \varrho(0) U_{\varphi}^{\dagger} \\
& =\sum_{n m} \int_{\mathbb{R}} d \varphi \frac{e^{-\varphi^{2} /\left(4 \Delta^{2}\right)}}{\sqrt{4 \pi \Delta^{2}}} e^{-i \varphi(n-m)} \varrho_{n m}(0)|n\rangle\langle m| \\
& =\sum_{n m} e^{-\Delta^{2}(n-m)^{2}} \varrho_{n m}(0)|n\rangle\langle m| .
\end{aligned}
$$

The parameter $\Delta$ is related to the width of the Gaussian distribution of the random phase-shift by the relation $\sigma_{r n d}^{2}=$ $2 \Delta^{2}$ : As one may expect, the broader the Gaussian distribution, the higher is the phase-noise affecting the quantum state.

If we consider the mode initially prepared in a coherent state $|\alpha\rangle$ with real amplitude, the phase-diffused state is a non-Gaussian mixed state with density operator given by

$$
\varrho_{\Delta, \alpha}=e^{-|\alpha|^{2}} \sum_{n, m}^{\infty} \frac{\alpha^{n+m} e^{-\Delta^{2}(n-m)^{2}}}{\sqrt{n ! m !}}|n\rangle\langle m|,
$$

in the Fock basis.

We have evaluated numerically the $\mathrm{nG} \delta_{B}\left[\varrho_{\Delta, \alpha}\right]$ for different values of the noise parameter $\Delta$ and of the average number of photons $|\alpha|^{2}$. The results are shown in Fig. 9. In the left panel we report $\delta_{B}$ as a function of $\Delta$ and for different values of $\alpha$ : large values of $\mathrm{nG}$ are achieved and the more intense the initial coherent state, the more non-Gaussian is the output. For large values of the noise parameter the off-diagonal elements of the density matrix are completely destroyed and the $\mathrm{nG}$
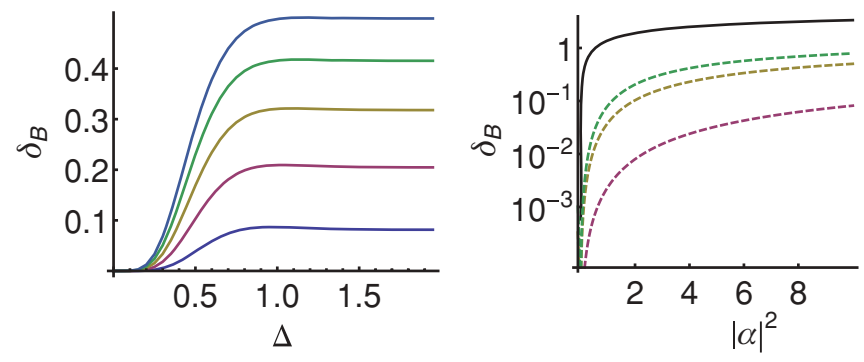

FIG. 9. (Color online) (Left) QRE-based $\mathrm{nG} \delta_{B}$ for coherent states undergoing phase-diffusion as a function of the noise parameter $\Delta$ and for different values of the average number of photons. From bottom to top, $|\alpha|^{2}=\{1,2,3,4,5\}$. (Right) QRE-based $\mathrm{nG} \delta_{B}$ for coherent states undergoing phase diffusion as a function of the average number of photons $|\alpha|^{2}$ for different values noise parameter: dashed lines, from bottom to top, $\Delta=\{0.25,0.5, \infty\}$. The black solid line represents the maximum value of $\mathrm{nG}$ at fixed number of photons.

approaches its asymptotic value, corresponding to the $\mathrm{nG}$ of a diagonal mixture of Fock states with a Poissonian distribution. The asymptotic value may be evaluated using Eq. (45) and choosing $q_{n}$ as a Poissonian distribution with mean value $\lambda=|\alpha|^{2}$. Before approaching the asymptotic value, $\delta_{B}\left[\varrho_{\Delta, \alpha}\right]$ is not monotone: A not much pronounced maximum of $\delta_{B}$ may be seen for intermediate values of $\Delta$. In the right panel of Fig. 9 we plot the $\mathrm{nG}$ for different values of $\Delta$ as a function of the number of photons $|\alpha|^{2}$. As we noticed earlier, the maximum value of $\delta_{B}\left[\varrho_{\Delta, \alpha}\right]$ at fixed $|\alpha|^{2}$ is not the asymptotic value. However, the difference is very small and thus, upon observing the behavior for different values of $\Delta$, we conclude that the maximum $\mathrm{nG}$ cannot be achieved by this family of quantum states.

\section{Kerr interaction}

One of the simplest unitary non-Gaussian evolutions is provided by the so-called self-Kerr effect taking place in third-order nonlinear $\chi^{(3)}$ media. The interaction Hamiltonian is given by

$$
H_{\mathrm{kerr}}=\Gamma\left(a^{\dagger} a\right)^{2}
$$

and the evolution operator by $U_{\text {kerr }}=\exp \left\{-i \gamma\left(a^{\dagger} a\right)^{2}\right\}$, where $\gamma=\Gamma t$ is a dimensionless coupling constant. Kerr interaction has been suggested to realize quantum nondemolition measurements, to enhance quantum estimation performances in quantum optics and to generate quantum superpositions [111, 112], as well as squeezing [113] and entanglement [52,53]. A known example of Kerr medium is provided by optical fibers where, however, nonlinearities are small and accompanied by other unwanted effects. Recently, larger Kerr nonlinearities have been proposed in many different physical systems [114] and have been observed with electromagnetically induced transparency [115], with Bose-Einstein condensates [116], and with cold atoms [117]. These results renewed the interest for the quantum effects of Kerr interaction, which are always accompanied by the generation of $\mathrm{nG}$. In the following, we consider the $\mathrm{nG}$ features of an initial coherent state $|\alpha\rangle$ 


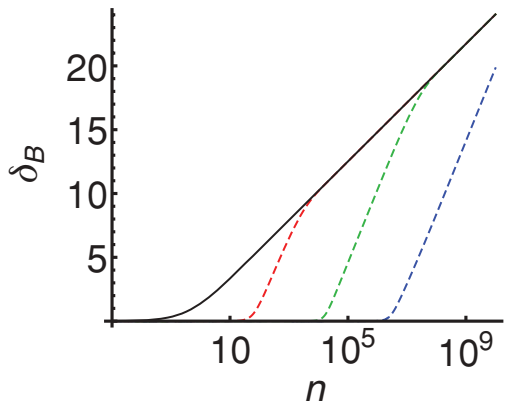

FIG. 10. (Color online) QRE-based $\mathrm{nG} \delta_{B}$ of coherent states undergoing Kerr interaction as a function of the average number of photons and for different values of the coupling constant $\gamma$. Dashed lines from left to right $\gamma=\left\{10^{-6}, 10^{-4}, 10^{-2}\right\}$. The black solid line is the maximum $\mathrm{nG}$ at fixed number of photons.

undergoing Kerr interaction,

$$
\left|\alpha_{\gamma}\right\rangle=U_{\mathrm{kerr}}|\alpha\rangle=e^{-|\alpha|^{2} / 2} \sum_{n=0}^{\infty} \frac{\alpha^{n}}{\sqrt{n !}} e^{-i \gamma n^{2}}|n\rangle .
$$

Since the evolution is unitary, the output state is still pure and the evaluation of the $\mathrm{nG} \delta_{B}$ is straightforward and involves only the computation of the covariance matrix of the evolved state. Non-Gaussianity $\delta_{B}\left[\left|\alpha_{\gamma}\right\rangle\right]$ is plotted in Fig. 10 as a function of the average number of photons $n=\left|\alpha^{2}\right|$ and for different values of the coupling constant $\gamma$. The maximum $\mathrm{nG}$ achievable for a given number of photons is also reported for comparison. As expected, $\mathrm{nG}$ is an increasing function of the initial energy $n$ and, for the range of values here considered, of the the coupling constant $\gamma$.

For $\gamma \approx 10^{-2}$ the maximum $\mathrm{nG}$ achievable at fixed energy is quite rapidly achieved, while for more realistic values of the Kerr coupling $\mathrm{nG}$ is obtained only for large values of the average number of photons. In the experiments proposed to obtain entanglement via the Kerr interaction [52,53], pulses with an average number up to $10^{8}$ photons are needed to compensate for the small nonlinearities of standard glass fibers. Therefore, in these regimes, the generation of entanglement is always accompanied by a large degree of $\mathrm{nG}$.

\section{NON-GAUSSIANITY AND DISTILLATION OF ENTANGLEMENT}

Long-distance quantum communication requires that the communicating parties share highly entangled states over a long distance. One has therefore to deal with the daunting task of distributing highly entangled states over long distances, overcoming losses and decoherence due to the unavoidable coupling of the system with the environment. For discretevariable states (qudit) entanglement-distillation protocols, where a subset of states with an high degree of entanglement are extracted from an enemble of less entangled states, have been proposed and experimentally demonstrated. As regards $\mathrm{CV}$ entanglement, it has been proved that entanglement distillation cannot be performed within the Gaussian world, that is, by starting from Gaussian entangled states and by linear optical components, homodyne detection, and classical communication. Non-Gaussianity is a necessary ingredient in an entanglement-distillation protocol. In particular, two different main approaches can be adopted: In the first approach, for example, the protocol of $[47,48]$, one starts with an entangled non-Gaussian state and then uses Gaussian operations based on linear optical elements, homodyne detection, or vacuum projective measurements. In the second approach one starts with an entangled Gaussian beam and tries to increase its entanglement by using non-Gaussian operations such as photon number conditional measurements. In [25-27] it has been proved that two-mode squeezed states with photons subtracted on the two modes can be used to obtain better teleportation fidelities. A first full scheme of entanglement distillation based on this idea has been presented in [49], where increase of entanglement by means of local photons subtraction from two-mode Gaussian states has been observed. In the following we review the protocols of [47] and [49] point out the role played by $\mathrm{nG}$ and its amount in the success of the protocols.

The protocol proposed in [47], from now on the B protocol, makes use of beam splitters and on/off detectors, that is, detectors only able to distinguish the presence or the absence of photons. The input state of the protocol is the state $\varrho \otimes \varrho$, that is, two replicas of a two-mode non-Gaussian. The two copies are mixed in a balanced beam splitter and then two of the output modes are directed into on/off photon detectors. The state is kept if both the local detectors are registering the outcome "zero," that is, at least in ideal conditions, the presence of the vaccum state. In [47] it has been proved that the B protocol drives the initial state toward a zero-displacement Gaussian state, which in turn constitute the only fixed points of the map. Moreover, under some assumptions on the initial state, the protocol acts as a purifying protocol as well, distilling the initial non-Gaussian entangled mixed state into a pure Gaussian entangled state. Notice that despite the fact that the output state of each step is obtained via (Gaussian) passive linear operations and Gaussian measurements (the projection on the vacuum state), the map cannot be described by a proper completely positive Gaussian operation [118].

As an illustrative example, let us consider the B protocol applied to the non-Gaussian pure state with the following nonzero matrix elements in the Fock basis $\varrho(a, b, c, d)=$ $\langle a, b|\varrho| c, d\rangle$ :

$$
\begin{gathered}
\varrho_{a}(0,0,0,0)=\frac{1}{1+\lambda^{2}}, \\
\varrho_{a}(0,0,1,1)=\varrho_{a}(1,1,0,0)=\frac{\lambda}{1+\lambda^{2}}, \\
\varrho_{a}(1,1,1,1)=\frac{\lambda^{2}}{1+\lambda^{2}} .
\end{gathered}
$$

In Fig. 11 we show the nG quantified by $\delta_{B}$ as a function of the parameter $\lambda$ for states obtained after different number of steps of the protocol. As a matter of fact, at fixed $\lambda, \mathrm{nG}$ is not always monotonically decreasing under the iteration of the protocol, and for $\lambda \approx 1$ the value of nG may increase, even achieving very high values. On the other hand, the overall effectiveness of the protocol is confirmed by our measure, since the range of values of $\lambda$ for which $\delta_{B} \approx 0$ increases at each step of the protocol. Overall, the use of our $\mathrm{nG}$ measure may help to tailor the distillation protocol depending on the initial conditions. 


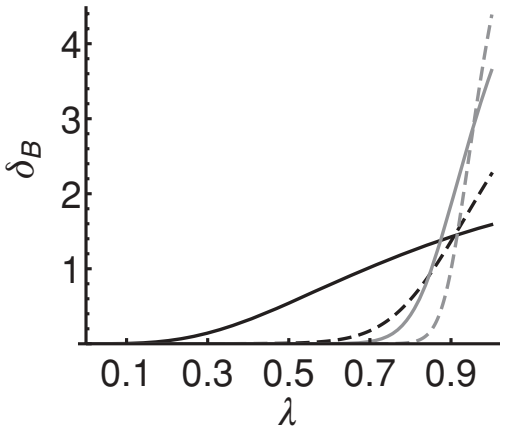

FIG. 11. Non-Gaussianity in the B protocol. The plot shows the QRE-based $\mathrm{nG} \delta_{B}$ of the output state after $s$ steps of the B protocol, when the initial state $\varrho_{a}$ is given in Eq. (60), as a function of the parameter $\lambda$. Step: $s=0$, black solid line; $s=5$, black dashed line; $s=10$, gray solid line; $s=20$, gray dashed line.

Let us now consider as initial states the pure state $\varrho_{a}$ of Eq. (60) and the mixed state $\varrho_{b}$ with nonzero matrix elements:

$$
\begin{gathered}
\varrho_{b}(0,0,0,0)=\frac{1}{1+\lambda^{2}}, \\
\varrho_{b}(0,0,1,1)=\varrho_{b}(1,1,0,0)=\frac{\lambda}{2\left(1+\lambda^{2}\right)}, \\
\varrho_{b}(1,1,1,1)=\frac{\lambda^{2}}{1+\lambda^{2}} .
\end{gathered}
$$

Both states converge toward pure Gaussian entangled states, and it has been shown [47] that entanglement increases at each step of the protocol. Here we investigate how much the gained entanglement is related to the $\mathrm{nG}$ of the initial state. For this purpose, since both entanglement and $\mathrm{nG}$ are increasing quantities with the number of photons, we consider a renormalized version of $\mathrm{nG} \delta_{R}[\varrho]$ and the relative entanglement gain at each step $\Delta^{(i)}$. The maximum amount of nG for a two-mode state with $N=\operatorname{Tr}\left[\varrho\left(a^{\dagger} a+b^{\dagger} b\right)\right]$ photons is

$$
\delta_{M}^{(2)}(N)=2[(1+N / 2) \ln (1+N / 2)-(N / 2) \ln (N / 2)],
$$

and the renormalized $\mathrm{nG}$ is defined as

$$
\delta_{R}[\varrho]=\frac{\delta_{B}[\varrho]}{\delta_{M}^{(2)}(N)} .
$$

We define the relative entanglement gain at the step $i$ as

$$
\Delta^{(i)}=\frac{E_{N}\left(\varrho^{(i)}\right)-E_{N}\left(\varrho^{(0)}\right)}{E_{N}\left(\varrho^{(0)}\right)},
$$

where $\varrho^{(i)}$ is the output state at the $i$ th step of the protocol. The degree of entanglement is quantified in terms of the logarithmic negativity, that is,

$$
E_{N}(\varrho)=\ln \left\|\varrho^{\Gamma}\right\|_{1},
$$

where \|\|$_{1}$ denotes the trace-norm and $\varrho^{\Gamma}$ is the partial transpose of $\varrho$ [119]. In Fig. 12 we plot for both states $\varrho_{a}$ and $\varrho_{b}$, the increase of entanglement $\Delta^{(i)}$ as a function of the renormalized $\mathrm{nG} \delta_{R}[\varrho]$. We observe that the more non-Gaussian the initial state, the larger is the entanglement increase at each step of the protocol. Therefore, we may say
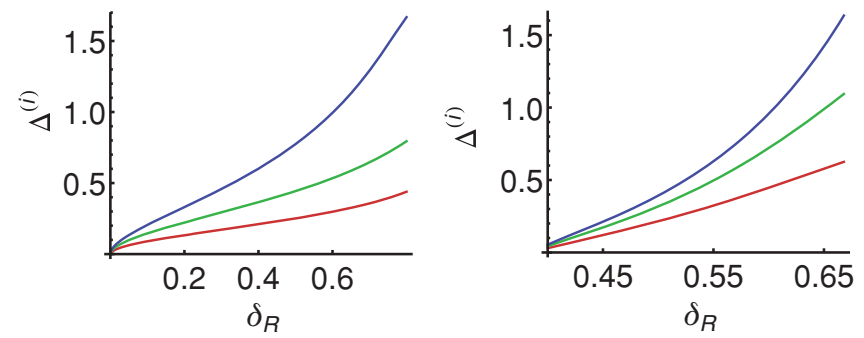

FIG. 12. (Color online) The relative increase of entanglement $\Delta^{(i)}$ at the $i$ th step of the B protocol as a function of the renormalized $\mathrm{nG}$ of the initial state (left, $\varrho_{A}$; right, $\varrho_{B}$ ). From bottom to top, $i=$ $\{1,2,5, \infty\}$

that at least for this particular protocol $\mathrm{nG}$ plays a relevant role, and it is quantitatively responsible for the good performances of the protocol.

Let us now consider the distillation protocol proposed in [49], from now on the $\mathrm{T}$ protocol. In this protocol, an entangled Gaussian state is obtained by mixing a single-mode squeezed state $|\psi\rangle=S(r)|0\rangle$ with the vacuum in a balanced beam splitter. Then, both the modes are split to perform a photon-subtraction and the state is kept if one or both detectors clicks (see [49] for details). The two possible output states of the distillation procedure can be written in a compact form as

$$
\begin{aligned}
\left|\psi_{\text {out }}^{(i)}\right\rangle_{A B} & =\mathcal{N} a_{A}^{n_{A}} a_{B}^{n_{B}} \hat{B}_{A B}(\pi / 4) \hat{S}_{A}(r)|0\rangle_{A}|0\rangle_{B} \\
& =\mathcal{N} \hat{B}_{A B}(\pi / 4) a_{A}^{n_{A}+n_{B}} \hat{S}_{A}(r)|0\rangle_{A}|0\rangle_{B},
\end{aligned}
$$

where if $\left(n_{A}, n_{B}\right)=(1,0)=(0,1)$ we have a single-photon subtracted state $\left|\psi_{\text {out }}^{(1)}\right\rangle$, while for $\left(n_{A}, n_{B}\right)=(1,1)$ we obtain the two-photon subtracted state $\left|\psi_{\text {out }}^{(2)}\right\rangle$. The non-Gaussianities of the two states can be easily evaluated by using the properties of the measure $\delta_{B}$, and in particular by exploiting the invariance under Gaussian unitary operation such as beamsplitter evolution and squeezing. After some calculations, it turns out that

$$
\begin{gathered}
\delta_{B}\left[\left|\psi_{\text {out }}^{(1)}\right\rangle\right]=\delta_{B}[|1\rangle], \\
\delta_{B}\left[\left|\psi_{\text {out }}^{(2)}\right\rangle\right]=\delta_{B}\left[\mathcal{N}^{\prime}(\mu|0\rangle+\sqrt{2} \nu|2\rangle)\right],
\end{gathered}
$$

where $\mu=\cosh (r), v=\sinh (r)$, and $\mathcal{N}^{\prime}$ is a normalization factor. We can immediately observe that for $\left|\psi_{\text {out }}^{(1)}\right\rangle$ the $\mathrm{nG}$ does not depend on the squeezing parameter $r$. In Fig. 13 we plot as a function of $r$ in the left panel the entanglement and in the right panel the non-Gaussianities of the two possible output states. We observe that for small values of $r$ the entanglement and the $\mathrm{nG}$ of the output states have a similar beahvior. However, this similarity is lost when we consider higher values of the squeezing; that is, we can say nothing in general about the relationship between the distilled entanglement and the $\mathrm{nG}$ for this protocol.

In summary, the role of the amount of $\mathrm{nG}$ in $\mathrm{CV}$ entanglement-distillation protocols is still not fully understood and worth studying for protocols employing non-Gaussian entangled states as an initial resource. In particular, an interesting open question that arises in this framework is whether there is a maximum amount of distillable entanglement at fixed $\mathrm{nG}$ of the resource state. 

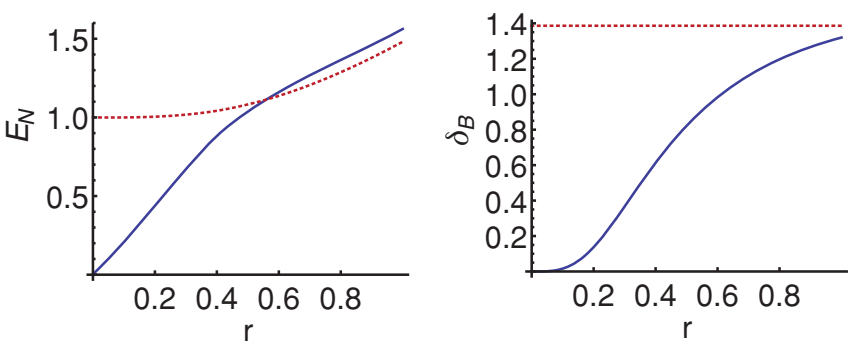

FIG. 13. (Color online) Entanglement-based (left) and QREbased $\mathrm{nG}$ (right) of the distilled states in the T protocol as a function of the squeezing parameter $r$. Dotted red line, one-photon subtracted state $\left|\psi_{\text {out }}^{(1)}\right\rangle$; solid blue line, two-photon subtracted state $\left|\psi_{\text {out }}^{(2)}\right\rangle$.

\section{NON-GAUSSIANITY AND QUANTUM COMMUNICATION}

As anticipated in the Introduction, Gaussian states play a relevant role in quantum communication, in particular for being extremal, at fixed covariance matrix for several relevant quantities [4]. For example, channel capacities are maximized by Gaussian states, whereas most of the entanglement measures are minimized by Gaussian states. In this section we prove the extremality conditions for some of these quantities by using our $\mathrm{nG}$ measure $\delta_{B}$ and studying the role played by $\mathrm{nG}$ itself in these inequalities

As a first example, let us consider a very important and well-known quantity in quantum information, that is, the $\chi$-Holevo quantity. Let us suppose to transmit information by encoding the symbols $a_{i}$, chosen with probabilities $p_{i}$ from an alphabet $\mathcal{A}$, in a set of quantum states $\varrho_{i}$. At each use of channel, the state preparation is described by the overall state $\varrho=\sum_{i} p_{i} \varrho_{i}$, and the Holevo quantity, which quantifies the amount of accessible information, is given by the formula $\chi(\varrho)=\mathrm{S}(\varrho)-\sum_{i} p_{i} \mathrm{~S}\left(\varrho_{i}\right)$. If we fix the covariance matrix of the overall state $\varrho$ and consider pure encoding states $\varrho_{i}$, we obtain (we denote $\delta_{B}=\delta$ in the rest of the paper)

$$
\chi(\varrho)=\mathrm{S}(\tau)-\delta[\varrho] .
$$

The maximum value of $\chi$ is attained by considering the Gaussian state and the $\mathrm{nG} \delta[\varrho]$ exactly quantifies how much information is lost by considering a non-Gaussian overall state.

Let us now consider bipartite states and rephrase the extremality condition for two quantities: the quantum mutual information and the quantum conditional entropy. The quantum mutual information, given a bipartite state $\varrho_{A B}$ is defined as $I(A: B)=\mathrm{S}\left(\varrho_{A}\right)+\mathrm{S}\left(\varrho_{B}\right)-\mathrm{S}\left(\varrho_{A B}\right)$ [120]. It quantifies the amount of correlations (classical and quantum) in a bipartite state and for pure states corresponds to the entanglement. In [121] it has been shown that at fixed covariance matrix $I(A: B)$ is minimized by Gaussian states. By using our measure $\delta$ the proof is simply based on the Lemma B5; that is,

$$
\begin{aligned}
I(A: B)= & \mathrm{S}\left(\varrho_{A}\right)+\mathrm{S}\left(\varrho_{B}\right)-\mathrm{S}\left(\varrho_{A B}\right) \\
= & \mathrm{S}\left(\tau_{A}\right)+\mathrm{S}\left(\tau_{B}\right)-\mathrm{S}\left(\tau_{A B}\right) \\
& +\left(\delta\left[\varrho_{A B}\right]-\delta\left[\varrho_{A}\right]-\delta\left[\varrho_{B}\right]\right) \\
\geqslant & I_{G}(A: B),
\end{aligned}
$$

where $I_{G}(A: B)=\mathrm{S}\left(\tau_{A}\right)+\mathrm{S}\left(\tau_{B}\right)-\mathrm{S}\left(\tau_{A B}\right)$ is the quantum mutual information obtained by considering the reference Gaussian states. The two mutual informations $I(A: B)$ and $I_{G}(A: B)$ differ exactly by the quantity

$$
\Delta_{2}=I(A: B)-I_{G}(A: B)=\delta\left[\varrho_{A B}\right]-\delta\left[\varrho_{A} \otimes \varrho_{B}\right] ;
$$

that is, the amount of correlations that are lost upon considering the Gaussian counterpart of $\varrho_{A B}$ is equal to the amount of $\mathrm{nG}$ that is lost by considering the tensor product of the partial states $\varrho_{A} \otimes \varrho_{B}$ instead of the (correlated) bipartite state $\varrho_{A B}$.

Another quantity that is known to be maximized, at fixed covariance matrix, by Gaussian states is the conditional entropy [108]. Conditional entropy is defined, given a bipartite state $\varrho_{A B}$ as $\mathrm{S}(A \mid B)=\mathrm{S}\left(\varrho_{A B}\right)-\mathrm{S}\left(\varrho_{B}\right)$ [120]. The proof of the extremality can be easily obtained by means of our QRE-based measure in the following way:

$$
\begin{aligned}
\mathrm{S}(A \mid B) & =\mathrm{S}\left(\varrho_{A B}\right)-\mathrm{S}\left(\varrho_{B}\right) \\
& =\mathrm{S}\left(\tau_{A B}\right)-\mathrm{S}\left(\tau_{A}\right)-\left(\delta\left[\varrho_{A B}\right]-\delta\left[\varrho_{B}\right]\right) \\
& \leqslant \mathrm{S}_{G}(A \mid B),
\end{aligned}
$$

where we used the monotonicity of the measure under partial trace, and where we defined the Gaussian conditional entropy $\mathrm{S}_{G}(A \mid B)=\mathrm{S}\left(\tau_{A B}\right)-\mathrm{S}\left(\tau_{B}\right)$ as the one evaluated by considering the reference Gaussian states. The two conditional entropies differ exactly by the quantity $\Delta_{1}=\delta\left[\varrho_{A B}\right]-\delta\left[\varrho_{B}\right]$; thus, the more robust the $\mathrm{nG}$ of the overall state under discarding a subsystem, the more the difference between the two quantities $\mathrm{S}_{G}(A \mid B)$ and $\mathrm{S}(A \mid B)$ is near to zero. This extremality condition is important for several reasons: the negative of the conditional entropy is a lower bound for the distillable entanglement, and thus, one can evaluate, given a generic bipartite state $\varrho_{A B}$, a simple lower bound on the distillable entanglement based only on first and second moments of the state. Moreover, according to the operational meaning of QRE given in [122] in the framework of quantum state merging, we observe that at fixed covariance matrix, it is always more convenient to use the non-Gaussian state. Indeed, for positive values, the quantum conditional entropy quantifies how much quantum information Alice needs to send to Bob so that he gains the full knowledge of the bipartite state $\varrho_{A B}$ given his previous knowledge about the partial state $\varrho_{B}$, while for negative values it turns out that Alice needs to send only classical information and moreover the two users gain entanglement to perform, for example, teleportation. As a consequence, at fixed covariance matrix, by using a non-Gaussian state you have to send less information or, for negative values, you gain more entanglement; the gain is exactly given by the degradation of the $\mathrm{nG}$ under partial trace, that is, how much $\mathrm{nG}$ is lost from the initial state $\varrho_{A B}$ tracing out the first Hilbert space.

A different interpretation of the conditional entropy has been also given in [123], in the context of the so-called private quantum decoupling. It has been shown that, in the limit of infinitely many copies of the initial state $\varrho_{A B}$, the ineliminable correlations between the two parties are quantified by the negative of the conditional entropy. Again we have that, at fixed covariance matrix, the non-Gaussian state has more ineliminable quantum correlations than the corresponding 
Gaussian one and that the more $\mathrm{nG}$ is lost under partial trace operation, the more these quantum correlations are present.

The preceding results lead one to speculate about nonGaussian correlations which, being encoded in a larger set of degrees of freedom, are, at fixed covariance matrix, higher than the Gaussian ones. In particular, we have observed this nontrivial connection between the robustness of $\mathrm{nG}$ under partial trace and decoupling operations, as well as in the quantum correlations present in non-Gaussian bipartite states. Recently, arguments have been supporting the conjecture that at fixed energy Gaussian entanglement is most robust against noise in a Markovian Gaussian channel [124]. On the other hand, the same analysis has also shown that robustness of nonGaussian states is comparable with that of Gaussian states for sufficiently high energy of the states. This implies that in these regimes non-Gaussian resources can be exploited to improve quantum communication protocols over approximately the same distances.

\section{NON-GAUSSIANITY AND PARAMETER ESTIMATION}

In an estimation problem one tries to infer the value the of a parameter $\lambda$ by measuring a different quantity $X$, which is somehow related to $\lambda$. This often happens in quantum mechanics and quantum information where many quantities of interest, for example, entanglement $[125,126]$, do not correspond to a proper observable and should be estimated from the measurement of one or more observable quantities [127]. Given a set $\left\{\varrho_{\lambda}\right\}$ of quantum states parametrized by the value of the quantity of interest, an estimator $\hat{\lambda}$ for $\lambda$ is a real function of the outcomes of the measurements performed on $\varrho_{\lambda}$. The quantum Cramer-Rao theorem [128,129] establishes a lower bound for the variance $\operatorname{Var}(\lambda)$ of any unbiased estimator, that is, for the estimation precision,

$$
\operatorname{Var}(\lambda) \geqslant \frac{1}{M H(\lambda)},
$$

in terms of the number of measurements $M$ and the so-called quantum Fisher information, which captures the statistical distinguishability of the states within the set and itself, is proportional to the Bures distance between states corresponding to infinitesimally close values of the parameter, that is,

$$
\begin{aligned}
H(\lambda) & =4 d_{B}^{2}\left(\varrho_{\lambda+d \lambda}, \varrho_{\lambda}\right) \\
& =2 \sum_{n m} \frac{\left|\left\langle\psi_{m}\left|\partial_{\lambda} \varrho_{\lambda}\right| \psi_{n}\right\rangle\right|^{2}}{\varrho_{n}+\varrho_{m}},
\end{aligned}
$$

where we have used the eigenbasis $\varrho_{\lambda}=\sum_{n} \varrho_{n}\left|\psi_{n}\right\rangle\left\langle\psi_{n}\right|$.

In an estimation problem where the variation of a parameter affects the Gaussian character of the involved states, one may expect the amount of $\mathrm{nG}$ to play a role in determining the estimation precision. This is indeed the case: The $\mathrm{nG} \delta_{B}$ provides an upper bound to the quantum Fisher information at fixed covariance matrix. This is more precisely expressed by the following.

Theorem 1. If $\tau_{\lambda}$ is a Gaussian state and an infinitesimal variation of the value of $\lambda$ drives it into a state $\varrho_{\lambda+d \lambda}$ with the same covariance matrix, then the $\mathrm{nG} \delta_{B}\left[\varrho_{\lambda+d \lambda}\right]$ provides an upper bound to the quantum Fisher information.
Proof. If $\varrho_{\lambda+d \lambda}$ and $\tau_{\lambda}$ have the same CM then the nG of $\varrho_{\lambda+d \lambda}, \quad \delta_{B}\left[\varrho_{\lambda+d \lambda}\right]=\mathrm{S}\left(\varrho_{\lambda+d \lambda} \| \tau_{\lambda}\right)$ equals the socalled Kubo-Mori-Bogolubov information $\widetilde{H}(\lambda)$ [130,131], which itself provides an upper bound for the quantum Fisher information $H(\lambda) \leqslant \widetilde{H}(\lambda)$ [132], thus proving the theorem.

The theorem says that the more non-Gaussian is the perturbed state, the more it may be distinguishable from the original one, thus allowing a more precise estimation. One may wonder that when $\varrho_{\lambda+d \lambda}$ is itself a Gaussian state $\tau_{\lambda+d \lambda}$ the theorem requires $H(\lambda)=0$; that is, no reliable estimation is possible. Indeed, this should be the case, since Gaussian states are uniquely determined by the first two moments and thus the requirement of having the same covariance matrix implies that $\tau_{\lambda+d \lambda}$ and $\tau_{\lambda}$ are actually the same quantum state.

For situations where the CM is changed by the perturbation, we have no general results. On the other hand, it has already been shown that non-Gaussian states improve quantum estimation of displacement and squeezing parameters [31] and of the loss parameter [32] compared to optimal Gaussian probes.

\section{EXPERIMENTALLY FRIENDLY LOWER BOUNDS TO QRE NG}

A drawback of the $\mathrm{nG}$ measure $\delta_{B}$ is that its evaluation requires the knowledge of the full density matrix $\varrho$. For this reason, it is often hard to compute when one has only partial information coming from some, maybe inefficient, measurements. In the literature different approaches have been proposed to estimate squeezing [133] and entanglement [80] of Gaussian and non-Gaussian states when only certain measurement are available in the laboratory. In the following we derive some lower bounds to the QRE-based $\mathrm{nG}$ measure for some classes of states by considering the possibility to perform on the states only certain efficient or inefficient measurements.

\section{A. Diagonal state and inefficient photodetection}

Let us consider a generic single-mode state diagonal in the Fock basis $\varrho=\sum_{n} p_{n}|n\rangle\langle n|$. Its $\mathrm{nG}$ can be evaluated as

$$
\delta[\varrho]=\mathrm{S}\left(v_{N}\right)-\mathrm{S}(\varrho)=\mathrm{S}\left(v_{N}\right)-H\left(p_{n}\right),
$$

where $v_{N}$ is a thermal state with the same average photon number $N=\sum_{n} n p_{n}$, and from now on

$$
H\left(p_{n}\right)=-\sum_{n} p_{n} \ln p_{n}
$$

denotes the Shannon entropy corresponding to the distribution $\left\{p_{n}\right\}$.

Let us consider now an inefficient photodetector described by POVM operators,

$$
\Pi_{m}=\sum_{s=m}^{\infty} \alpha_{m, s}(\eta)|s\rangle\langle s|,
$$

with $\alpha_{m, s}(\eta)$ defined in Eq. (51) and where $\eta$ is the efficiency of the detector. Using this kind of detection one can reconstruct 
the probability distribution $q_{m}=\operatorname{Tr}\left[\varrho \Pi_{m}\right]$. We want to show that the quantity

$$
\epsilon_{A}[\varrho]=\mathrm{S}\left(v_{M}\right)-H\left(q_{m}\right)
$$

with $M=\sum_{m} m q_{m}=\eta N$, is a lower bound on the actual $\mathrm{nG}$ $\delta[\varrho]$. To this aim we remind that an inefficient photodetection can be described by mixing the quantum state $\varrho$ with a vacuum state at a beam splitter with transmissivity $\eta$ followed by a perfect photodetection with projective operators $P_{m}=$ $|m\rangle\langle m|$. The corresponding probability distribution is therefore

$$
\begin{aligned}
q_{m} & =\operatorname{Tr}_{12}\left[U_{B S}(\eta) \varrho \otimes|0\rangle\left\langle 0\left|U_{B S}^{\dagger}(\eta)\right| m\right\rangle\langle m| \otimes \mathbb{1}\right] \\
& =\operatorname{Tr}_{1}[\mathcal{E}(\varrho)|m\rangle\langle m|]
\end{aligned}
$$

where $\mathcal{E}(\varrho)=\operatorname{Tr}_{2}\left[U_{B S}(\eta) \varrho \otimes|0\rangle\langle 0| U_{B S}^{\dagger}(\eta)\right]$ is the loss channel applied on the quantum state $\varrho$. Since $\varrho$ is diagonal in the Fock basis we can easily show that $\mathcal{E}(\varrho)$ is still diagonal,

$$
\mathcal{E}(\varrho)=\sum_{n} p_{n} \mathcal{E}(|n\rangle\langle n|)=\sum_{n} \sum_{l=0}^{n} p_{n} \alpha_{l, n}(\eta)|l\rangle\langle l|,
$$

where we used that $\mathcal{E}(|n\rangle\langle n|)=\sum_{l=0}^{n} \alpha_{l, n}(\eta)|l\rangle\langle l|$. By performing an efficient photodetection we can experimentally obtain the probability distribution

$$
q_{m}=\operatorname{Tr}[\mathcal{E}(\varrho)|m\rangle\langle m|]=\sum_{n=m}^{\infty} p_{n} \alpha_{m, n}(\eta) .
$$

The quantum state $\mathcal{E}(\varrho)$ in fully described by $q_{m}$ and by observing Eq. (72) and (73) we can easily see that $\epsilon_{A}[\varrho]=$ $\delta[\mathcal{E}(\varrho)]$. By simply using the fact the $\mathrm{nG}$ measure $\delta[\varrho]$ is nonincreasing under Gaussian maps, such as $\mathcal{E}$, we finally obtain

$$
\epsilon_{A}[\varrho]=\delta[\mathcal{E}(\varrho)] \leqslant \delta[\varrho]
$$

This inequality tells us that by performing an inefficient photodetection on a given quantum state $\varrho$ diagonal in the Fock basis, i.e. by measuring $q_{m}$, one can use Eq. (73) to obtain a lower bound on the actual $\mathrm{nG} \delta[\varrho]$.

\section{B. State with a thermal reference Gaussian state and ideal photodetection}

Let us consider a quantum state $\varrho=\sum_{n, m} p_{n, m}|n\rangle\langle m|$ such that $\operatorname{Tr}[\varrho a]=\operatorname{Tr}\left[\varrho a^{2}\right]=0$. The corresponding reference Gaussian state is a thermal state $v_{N}$ with the same average number of photons $N=\operatorname{Tr}\left[\varrho a^{\dagger} a\right]$. Let us consider now the quantum state $\left.\varrho\right|_{d}$ obtained considering only the photon number distribution $p_{n, n}$ and removing all the off-diagonal elements, that is, $\left.\varrho\right|_{d}=\sum_{n} p_{n, n}|n\rangle\langle n|$. The reference Gaussian state of $\left.\varrho\right|_{d}$ is again the thermal state $v_{N}$. Let us consider now the state

$$
\mathcal{N}_{\Delta}(\varrho)=\sum_{n, m} e^{-\Delta^{2}(n-m)^{2}} p_{n, m}|n\rangle\langle m|,
$$

which physically corresponds to a phase diffusion applied to the initial state. As a matter of fact, the same kind of evolved state may be obtained by the application of a random zeromean Gaussian distributed phase shift to $\varrho$, that is,

$$
\mathcal{N}_{\Delta}(\varrho)=\int_{R} d \phi \frac{e^{-\phi^{2} /\left(4 \Delta^{2}\right)}}{\sqrt{4 \pi \Delta^{2}}} U_{\phi} \varrho U_{\phi}^{\dagger},
$$

where $U_{\phi}=\exp \left\{-i a^{\dagger} a \phi\right\}$. Upon using the invariance under unitary operators and the concavity of the von Neumann entropy, one may show that

$$
\mathrm{S}\left(\mathcal{N}_{\Delta}(\varrho)\right) \geqslant \int_{R} d \phi \frac{e^{-\phi^{2} /\left(4 \Delta^{2}\right)}}{\sqrt{4 \pi \Delta^{2}}} \mathrm{~S}\left(U_{\phi} \varrho U_{\phi}^{\dagger}\right)=\mathrm{S}(\varrho) .
$$

From Eq. (76) we have that $\left.\varrho\right|_{d}=\lim _{\Delta \rightarrow \infty} \mathcal{N}_{\Delta}(\varrho)$ and because of Eq. (78) we arrive at

$$
H\left(p_{n, n}\right)=\mathrm{S}\left(\left.\varrho\right|_{d}\right) \geqslant \mathrm{S}(\varrho)
$$

It is straightforward to see that the $\mathrm{nG}$ of $\varrho$ is lower bounded by the $\mathrm{nG}$ evaluated by considering only the photon-number distribution of the state, that is,

$$
\epsilon_{B}[\varrho]=\mathrm{S}\left(v_{N}\right)-H\left(p_{n, n}\right) \leqslant \mathrm{S}\left(v_{N}\right)-\mathrm{S}(\varrho)=\delta[\varrho] .
$$

\section{State with a thermal reference Gaussian state and inefficient photodetection}

Let us take a quantum state $\varrho$ as in the previous section, but considering the case of an inefficient photodetection. From the measurement, we obtain the distribution $q_{m}=\operatorname{Tr}\left[\varrho \Pi_{m}\right]$, and we can thus define a quantum state

$$
\theta_{M}=\sum_{m} q_{m}|m\rangle\langle m|
$$

having $M=\sum_{m} m q_{m}$ average photons. As in Sec. XA, we have $\theta_{M}=\mathcal{E}\left(\left.\varrho\right|_{d}\right)$, with $\left.\varrho\right|_{d}$ defined as before and $\mathcal{E}$ denoting the loss channel. Then, by using the monotonicity of $\mathrm{nG}$ under Gaussian maps, and the previous results, we obtain

$$
\epsilon_{C}[\varrho]=\mathrm{S}\left(v_{M}\right)-H\left(q_{m}\right)=\delta\left[\theta_{M}\right] \leqslant \delta\left[\left.\varrho\right|_{d}\right] \leqslant \delta[\varrho]
$$

\section{Generic state with known covariance matrix and ideal photodetection}

Let us consider a generic single-mode state $\varrho=$ $\sum_{n, m} p_{n, m}|n\rangle\langle m|$ with a reference Gaussian state $\tau$. Its $\mathrm{nG}$ is evaluated as

$$
\delta[\varrho]=\mathrm{S}(\tau)-\mathrm{S}(\varrho)
$$

Let us consider the quantum state $\left.\varrho\right|_{d}=\sum_{n} p_{n, n}|n\rangle\langle n|$ as in the previous case and suppose that we are able to evaluate its covariance matrix of $\varrho$ (and thus the entropy of the reference Gaussian state $\tau)$. We proved before that $H\left(p_{n, n}\right)=\mathrm{S}\left(\left.\varrho\right|_{d}\right) \geqslant$ $\mathrm{S}(\varrho)$ and thus we have the computable lower bound on the $\mathrm{nG}$ :

$$
\epsilon_{D}[\varrho]=\mathrm{S}(\tau)-H\left(p_{n, n}\right) \leqslant \delta[\varrho]
$$




\section{E. Generic state with inefficient photodetection}

Let us consider a generic single-mode state $\varrho=$ $\sum_{n, m} p_{n, m}|n\rangle\langle m|$ with a reference Gaussian state $\tau$. Because of the monotonicity of the measure under Gaussian maps, we have

$$
\delta[\varrho] \geqslant \delta[\mathcal{E}(\varrho)]=\mathrm{S}\left(\tau_{\eta}\right)-\mathrm{S}(\mathcal{E}(\varrho)),
$$

where $\tau_{\eta}=\mathcal{E}(\tau)$. Again, by using the inequality derived in Eq. (79) we obtain

$$
\epsilon_{E}[\varrho]=\mathrm{S}\left(\tau_{\eta}\right)-H\left(q_{m}\right) \leqslant \delta[\varrho],
$$

where $q_{m}=\operatorname{Tr}\left[\varrho \Pi_{m}\right]$. This general lower bound can be useful when the covariance matrix of the state can be easily derived from the photon number statistics of $\varrho$ (e.g., for phase-averaged coherent states).

\section{CONCLUSIONS}

Non-Gaussianity is a resource for quantum information processing and thus we urge a measure able to quantify the non-Gaussian character of a quantum state. In this paper we have addressed $\mathrm{nG}$ of states and operations in CV systems and have illustrated in detail two measures of $\mathrm{nG}$ proposed in [76,77], along with their properties and the relationships between them. We used them to assess some Gaussification and de-Gaussification processes, and in particular we studied the role of the amount of $\mathrm{nG}$ in two entanglement-distillation protocols proposed in the literature. The role of $\mathrm{nG}$ appears to depend on the protocol itself, and at least in one of the two protocols the amount of gained entanglement at each step of the protocol is monotonous with the $\mathrm{nG}$ of the initial low-entangled state. We have also reconsidered the extremality of Gaussian states in terms of our measure based on the QRE, for some relevant quantities in quantum communication, such as conditional entropy, mutual information, and Holevo bound. In particular, we found that in the bipartite setting there is a, probably not entirely understood, connection between correlations and $\mathrm{nG}$ : At fixed covariance matrix non-Gaussian states have more correlations and this excess of correlations is related to the amount of $\mathrm{nG}$ that the quantum state loses under partial trace operation or under decoupling. These results, together with recent ones on the robustness of non-Gaussian entanglement in noisy Markovian channels implies that there are regimes where non-Gaussian resources can be exploited to improve quantum communication protocols. We have also seen that QRE $n G$ is a bound for the quantum Fisher information at fixed covariance matrix and thus the $\mathrm{nG}$ features of quantum states may also be used to improve parameter estimation with continuous variables. Finally, since the evaluation of the QRE $\mathrm{nG}$ measure requires the knowledge of the full density matrix, we derive some experimentally friendly lower bounds to $\mathrm{nG}$ for some classes of states and by considering the possibility of performing on the states only certain efficient or inefficient measurements.

Our analysis of the properties and the applications of the two measures of $\mathrm{nG}$ has shown that they provide a suitable quantification of $\mathrm{nG}$ for the purposes of quantum information. In particular, the QRE-based $\mathrm{nG} \delta_{B}$ has several operational characterizations and it is not too difficult to evaluate. Since all states $\varrho$ with the same first two moments at fixed purity have the same amount of $\mathrm{nG} \delta_{B}[\varrho]$, an interesting and remarkable picture emerges: For many purposes the effects of $\mathrm{nG}$ may be described by a single global parameter rather than ascribed to a specific higher moment. This is perhaps our main conclusion. Overall, our results suggest that in terms of resources for quantum information, the amount of $\mathrm{nG}$ of a quantum state can be evaluated using $\delta_{B}$, with $\delta_{A}$ serving as a fine-tuning tool for specific purposes.

There are several open problems requiring further investigation about $\mathrm{nG}$ of quantum states. Among them we mention the following ones, which provides a summary of the unanswered questions posed in our paper: (i) Is there any general relation between the two measures of $\mathrm{nG}$, at least for specific class of states? (ii) Is there a maximum amount of distillable entanglement at fixed $\mathrm{nG}$ ? (iii) What is the role of $\mathrm{nG}$ in parameter estimation involving a change in the covariance matrix?

In conclusion, $\mathrm{nG}$ is a resource that can be quantified. Our results pave the way for further development and suggest that a deeper understanding of the geometrical and analytical structures underlying the non-Gaussian features of states and operations could be a powerful tool for the effective implementation of quantum information processing with CVs.

\section{ACKNOWLEDGMENTS}

The authors thank Konrad Banaszek, Carmen Invernizzi, Stefano Olivares, Paolo Giorda, Michele Allegra, Ruggero Vasile, Sabrina Maniscalco, Marco Barbieri, Alberto Porzio, Salvatore Solimeno, Janika Paavola, Gerardo Adesso, Mauro Paternostro, Alessia Allevi, Maria Bondani, Francesca Beduini, Alessio Serafini, Jarda Rehacek, and Zdenel Hradil for useful discussions.
[1] A. Ferraro, S. Olivares, and M. G. A. Paris, Gaussian States in Quantum Information (Bibliopolis, Napoli, 2005).

[2] J. Eisert, and M. B. Plenio, Int. J. Quantum Inf. 1, 479 (2003).

[3] F. Dell'Anno, S. De Siena, and F. Illuminati, Phys. Rep. 428, 53 (2006).

[4] M. M. Wolf, G. Giedke, and J. I. Cirac, Phys. Rev. Lett. 96, 080502 (2006).

[5] F. Grosshans and N. J. Cerf, Phys. Rev. Lett. 92, 047905 (2004).

[6] M. Navascues, F. Grosshans, and A. Acin, Phys. Rev. Lett. 97, 190502 (2006).
[7] R. Garcia-Patron and N. J. Cerf, Phys. Rev. Lett. 97, 190503 (2006).

[8] S. L. Braunstein, and P. van Loock, Rev. Mod. Phys. 77, 513 (2005).

[9] J. Eisert, S. Scheel, and M. B. Plenio, Phys. Rev. Lett. 89, 137903 (2002).

[10] G. Giedke and J. I. Cirac, Phys. Rev. A 66, 032316 (2002).

[11] J. Fiurasek, Phys. Rev. Lett. 89, 137904 (2002).

[12] J. Niset, J. Fiurasek, and N. J. Cerf, Phys. Rev. Lett. 102, 120501 (2009). 
[13] T. C. Ralph, A. Gilchrist, G. J. Milburn, W. J. Munro, and S. Glancy, Phys. Rev. A 68, 042319 (2003).

[14] A. P. Lund, T. C. Ralph, and H. L. Haselgrove, Phys. Rev. Lett. 100, 030503 (2008).

[15] K. Banaszek and K. Wódkiewicz, Phys. Rev. A 58, 4345 (1998).

[16] K. Banaszek and K. Wódkiewicz, Phys. Rev. Lett. 82, 2009 (1999).

[17] H. Jeong, J. Lee, and M. S. Kim, Phys. Rev. A 61, 052101 (2000).

[18] R. Filip and L. Mista, Phys. Rev. A 66, 044309 (2002).

[19] Z.-B. Chen, J.-W. Pan, G. Hou, and Y.-D. Zhang, Phys. Rev. Lett. 88, 040406 (2002).

[20] H. Nha, and H. J. Carmichael, Phys. Rev. Lett. 93, 020401 (2004).

[21] C. Invernizzi, S. Olivares, M. G. A. Paris, and K. Banaszek, Phys. Rev. A 72, 042105 (2005).

[22] E. Pazy, A. Vardi, and Y. B. Band, Phys. Rev. Lett. 93, 120409 (2004); R. García-Patrón, J. Fiurášek, and N. J. Cerf, Phys. Rev. A 71, 022105 (2005).

[23] F. LeKien, S. Dutta Gupta, V. I. Balykin, and K. Hakuta, Phys. Rev. A 72, 032509 (2005).

[24] A. Ferraro and M. G. A. Paris, J. Opt. B: Quantum Semiclass. Opt. 7, 174 (2005).

[25] T. Opatrny, G. Kurizki, and D. G. Welsch, Phys. Rev. A 61, 032302 (2000).

[26] P. T. Cochrane, T. C. Ralph, and G. J. Milburn, Phys. Rev. A 65, 062306 (2002).

[27] S. Olivares, M. G. A. Paris, and R. Bonifacio, Phys. Rev. A 67, 032314 (2003).

[28] N. J. Cerf, O. Kruger, P. Navez, R. F. Werner, and M. M. Wolf, Phys. Rev. Lett. 95, 070501 (2005).

[29] F. Ferreyrol, M. Barbieri, R. Blandino, S. Fossier, R. TualleBrouri, and P. Grangier, Phys. Rev. Lett. 104, 123603 (2010).

[30] G. Y. Xiang, T. C. Ralph, A. P. Lund, N. Walk, and G. J. Pryde, Nature Photonics 4, 316 (2010).

[31] M. G. Genoni, C. Invernizzi, and M. G. A. Paris, Phys. Rev. A 80, 033842 (2009).

[32] G. Adesso, F. Dell' Anno, S. De Siena, F. Illuminati, and L. A. M. Souza, Phys. Rev. A 79, 040305(R) (2009).

[33] S. Olivares and M. G. A. Paris, J. Opt. B 7, S616 (2005).

[34] M. S. Kim, J. Phys. B 41, 133001 (2008).

[35] A. I. Lvovsky, H. Hansen, T. Aichele, O. Benson, J. Mlynek, and S. Schiller, Phys. Rev. Lett. 87, 050402 (2001).

[36] J. Wenger, R. Tualle-Brouri, and P. Grangier, Phys. Rev. Lett. 92, 153601 (2004).

[37] A. Zavatta, S. Viciani, and M. Bellini, Phys. Rev. A 70, 053821 (2004).

[38] A. Ourjoumtsev, R. Tualle-Brouri, J. Laurat, and P. Grangier, Science 312, 83 (2006).

[39] J. S. Neergard-Nielsen, B. M. Nielsen, C. Hettich, K. Molmer, and E. S. Polzik, Phys. Rev. Lett. 97, 083604 (2006).

[40] A. Ourjoumtsev, H. Jeong, R. Tualle-Brouri, and P. Grangier, Nature (London) 448, 784 (2007).

[41] A. Zavatta, V. Parigi, and M. Bellini, Phys. Rev. A 75, 052106 (2007).

[42] V. Parigi, A. Zavatta, M. S. Kim, and M. Bellini, Science 317, 1890 (2007).

[43] A. Zavatta, V. Parigi, M. S. Kim, H. Jeong, and M. Bellini, Phys. Rev. Lett. 103, 140406 (2009).
[44] A. Ourjoumtsev, F. Ferreyrol, R. Tualle-Brouri, and P. Grangier, Nat. Phys. 5, 189 (2009).

[45] A. Ourjoumtsev, A. Dantan, R. Tualle-Brouri, and P. Grangier, Phys. Rev. Lett. 98, 030502 (2007).

[46] A. Franzen, B. Hage, J. DiGuglielmo, J. Fiurasek, and R. Schnabel, Phys. Rev. Lett. 97, 150505 (2006).

[47] D. E. Browne, J. Eisert, S. Scheel, and M. B. Plenio, Phys. Rev. A 67, 062320 (2003); J. Eisert, D. E. Browne, S. Scheel, and M. B. Plenio, Ann. Phys. 311, 431 (2004).

[48] B. Hage, A. Samblowski, J. Di Gugliemo, A. Franzen, J. Fiurasek, and R. Schnabel, Nat. Phys. 4, 915 (2008).

[49] H. Takahashi, J. S. Neergaard-Nielsen, M. Takeuchi, M. Takeoka, K. Hayasaka, A. Furusawa, and M. Sasaki, Nature Photonics 4, 178 (2010).

[50] V. D'Auria, C. de Lisio, A. Porzio, S. Solimeno, J. Anwar, and M. G. A. Paris, Phys. Rev. A 81, 033846 (2010).

[51] A. Chiummo, M. De Laurentis, A. Porzio, S. Solimeno, and M. G. A. Paris, Opt. Express 13, 948 (2005).

[52] C. Silberhorn, P. K. Lam, O. Weiß, F. König, N. Korolkova, and G. Leuchs, Phys. Rev. Lett. 86, 4267 (2001).

[53] O. Glöckl, U. L. Andersen, and G. Leuchs, Phys. Rev. A 73, 012306 (2006).

[54] T. Tyc and N. Korolkova, New J. Phys. 10, 023041 (2008).

[55] M. G. A. Paris, M. Cola, and R. Bonifacio, Phys. Rev. A 67, 042104 (2003).

[56] G. M. D’Ariano, P. Kumar, C. Macchiavello, L. Maccone, and N. Sterpi, Phys. Rev. Lett. 83, 2490 (1999).

[57] S. Olivares, M. G. A. Paris, J. Opt. B 7, S392 (2005).

[58] D. T. Pegg, L. S. Phillips, and S. M. Barnett, Phys. Rev. Lett. 81, 1604 (1998).

[59] G. M. D'Ariano, L. Maccone, M. G. A. Paris, and M. F. Sacchi, Phys. Rev. A 61, 053817 (2000); Fort. Phys. 48, 511 (2000).

[60] Lu-Ming Duan, G. Giedke, J. I. Cirac, and P. Zoller, Phys. Rev. Lett. 844002 (2000).

[61] B. Hladky, G. Drobny, and V. Buzek, Phys. Rev. A 61, 022102 (2000).

[62] M. G. A. Paris, Phys. Rev. A 62, 033813 (2000).

[63] J. Clausen, M. Dakna, L. Knöll, and D.-G. Welsch, J. Opt. B 1, 322 (1999).

[64] M. G. A. Paris, Phys. Lett. A 217, 78 (1996).

[65] A. Napoli, A. Messina, and S. Maniscalco, Acta Phys. Slov. 50, 519 (2000).

[66] F. Plastina and F. Piperno, Eur. Phys. J. D 5, 411 (1999).

[67] M. Ban, Opt. Comm. 143, 225 (1997).

[68] A. Kozhekin, G. Kurizki, and B. Sherman, Phys. Rev. A 54, 3535 (1996).

[69] M. S. Kim, J. Phys. B 41, 133001 (2008).

[70] T. Gerrits, S. Glancy, T. S. Clement, B. Calkins, A. E. Lita, A. J. Miller, A. L. Migdall, S. W. Nam, R. P. Mirin, and E. Knill, Phys. Rev. A 82, 031802(R) (2010).

[71] M. Sasaki and S. Suzuki, Phys. Rev. A 73, 043807 (2006).

[72] M. G. Genoni, F. A. Beduini, A. Allevi, M. Bondani, S. Olivares, and M. G. A. Paris, Phys. Scr. T 140, 014007 (2010).

[73] A. Allevi, A. Andreoni, F. A. Beduini, M. Bondani, M. G. Genoni, S. Olivares, and M. G. A. Paris, Eur. Phys. Lett. 92, 20007 (2010).

[74] S. Deléglise, I. Dotsenko, C. Sayrin, J. Bernu, and M. Brune, J.-M. Raimond, and S. Haroche, Nature (London) 455, 510 (2008). 
[75] M. Hofheinz, E. M. Weig, M. Ansmann, R. C. Bialczak, E. Lucero, M. Neeley, A. D. O'Connell, H. Wang, J. M. Martinis, and N. Cleland, Nature (London) 454, 310 (2008).

[76] M. G. Genoni, M. G. A. Paris, and K. Banaszek, Phys. Rev. A 76, 042327 (2007).

[77] M. G. Genoni, M. G. A. Paris, and K. Banaszek, Phys. Rev. A 78, 060303(R) (2008).

[78] J. Solomon Ivan, M. Sanjay Kumar, and R. Simon, e-print arXiv:0812.2800v1.

[79] F. Dell'Anno, S. De Siena, and F. Illuminati, Phys. Rev. A 81, 012333 (2010).

[80] G. Adesso, Phys. Rev. A 79, 022315 (2009).

[81] F. Casagrande, A. Lulli, and M. G. A. Paris, Phys. Rev. A 75, 032336 (2007)

[82] M. Paternostro, G. Adesso, and S. Campbell, Phys. Rev. A 80, 062318 (2009).

[83] A. Mandilara, E. Karpov, and N. J. Cerf, Phys. Rev. A 79, 062302 (2009).

[84] R. L. Hudson, Rep. Math. Phys. 6, 249 (1974).

[85] A. Mandilara, E. Karpov, and N. J. Cerf, e-print arXiv:0910.3473.

[86] A. Allevi, A. Andreoni, M. Bondani, M. G. Genoni, and S. Olivares, Phys. Rev. A 82, 013816 (2010).

[87] X. Chen, Phys. Rev. D 72, 123518 (2005).

[88] G. I. Rigopoulos, E. P. S. Shellard, and B. J. W. van Tent, Phys. Rev. D 73, 083522 (2006).

[89] A. Bernui, C. Tsallis, and T. Villela, Europhys. Lett. 78, 19001 (2007).

[90] M. C. W. van Rossum, I. V. Lerner, B. L. Altshuler, and T. M. Nieuwenhuizen, Phys. Rev. B 55, 4710 (1997).

[91] P. Mohanty and R. A. Webb, Phys. Rev. Lett. 88, 146601 (2002).

[92] A. G. Huibers, S. R. Patel, C. M. Marcus, P. W. Brouwer, C. I. Duruoz, and J. S. Harris, Phys. Rev. Lett. 81, 1917 (1998).

[93] A. Hyvarinen and E. Oja, Neural Networks 13, 411 (2000).

[94] A. Hyvarinen, Adv. Neural Inf. Process. Syst. 10, 273 (1998).

[95] K. E. Cahill and R. J. Glauber, Phys. Rev. 177, 1882 (1969).

[96] J. Williamson, Am. J. Math. 58, 141 (1936).

[97] A. Serafini, F. Illuminati, and S. De Siena, J. Phys. B 37, L21 (2004).

[98] V. V. Dodonov, O. Manko, V. Manko, and A. Wunsche, J. Mod. Opt. 47, 633 (2000).

[99] V. V. Dodonov and M. B. Renò, Phys. Lett. A 308, 249 (2003).

[100] P. Marian, T. A. Marian, and H. Scutaru, Phys. Rev. A 69, 022104 (2004).

[101] M. G. Genoni and M. G. A. Paris, Int. J. Quantum Inf. 7, 97 (2009).

[102] V. Vedral, Rev. Mod. Phys. 74, 197 (2002).

[103] B. Schumacher and M. Westmoreland, American Mathematical Society Contemporary Mathematics, Series: Quantum Information and Quantum Computation (American Mathematical Society, Providence, 2002), p. 305.

[104] J. Rau, Phys. Lett. A 374, 3715 (2010).

[105] G. S. Agarwal, Phys. Rev. Lett. 57, 827 (1986); J. Opt. Soc. Am. B 5, 1940 (1988).

[106] V. C. Usenko and B. I. Lev, Phys. Lett. A 348, 17 (2005).
[107] V. C. Usenko and M. G. A. Paris, Phys. Rev. A 75, 043812 (2007); Phys. Lett. A 374, 1342 (2010).

[108] J. Eisert and M. M. Wolf, Quantum Information with Continous Variables of Atoms and Light, edited by N. J. Cerf, G. Leuchs, and E. S. Polzik (Imperial College Press, London, 2007), pp. 23-42.

[109] M. B. Plenio and S. Virmani, Quantum Inf. Comput. 7, 1 (2007).

[110] M. Ohia and D. Petz, Quantum Entropy and Its Use (Springer, Berlin, 1993).

[111] B. Yurke and D. Stoler, Phys. Rev. Lett. 57, 13 (1986); R. Loudon and P. L. Knight, J. Mod. Opt. 34, 709 (1987); A. D. Wilson-Gordon, V. Buzek, and P. L. Knight, Phys. Rev. A 44, 7647 (1991).

[112] M. G. A. Paris, J. Opt. B 1, 662 (1999).

[113] K. Sundar, Phys. Rev. A 53, 1096 (1996).

[114] A. K. Mohapatra, M. G. Bason, B. Butscher, K. J. Weatherill, and C. S. Adams, Nat. Phys. 4, 890 (2008); F. Brandao, M. Hartmann, and M. Plenio, New J. Phys. 10, 043010 (2008); E. Babourina-Brooks, A. Doherty, and G. J. Milburn, ibid. 10, 105020 (2008).

[115] A. Imamoglu, H. Schmidt, G. Woods, and M. Deutsch, Phys. Rev. Lett. 79, 1467 (1997); M. J. Werner and A. Imamoglu, Phys. Rev. A 61, 011801 (1999).

[116] L. V. Hau, S. E. Harris, Z. Dutton, and C. H. Behroozi, Nature (London) 397, 594 (1999).

[117] H. Kang and Y. Zhu, Phys. Rev. Lett. 91, 093601 (2003).

[118] As discussed in Lemma B6, the $\mathrm{nG}$ measure $\delta_{B}$ is monotone only under full CP Gaussian maps. In this case we are considering the Krauss operator corresponding to the vacuum outcomes of the measurement, and, even if only Gaussian operations and Gaussian measurements are involved, the inequality expressed in Lemma B6 can be violated.

[119] A. Peres, Phys. Rev. Lett. 77, 1412 (1996).

[120] N. J. Cerf and C. Adami, Phys. Rev. Lett. 79, 5194 (1997).

[121] A. S. Holevo and R. F. Werner, Phys. Rev. Lett. 63, 032312 (2004).

[122] M. Horodecki, J. Oppenheim, and A. Winter, Nature (London) 436, 673 (2005).

[123] F. Buscemi, New J. Phys. 11, 123002 (2010).

[124] M. Allegra, P. Giorda, and M. G. A. Paris, Phys. Rev. Lett. 105, 100503 (2010).

[125] M. G. Genoni, P. Giorda, and M. G. A. Paris, Phys. Rev. A 78, 032303 (2008).

[126] G. Brida, I. P. Degiovanni, A. Florio, M. Genovese, P. Giorda, A. Meda, M. G. A. Paris, and A. Shurupov, Phys. Rev. Lett. 104, 100501 (2010).

[127] M. G. A. Paris, Int. J. Quantum Inf. 7, 125 (2009).

[128] S. L. Braunstein and C. L. Caves, Phys. Rev. Lett. 72, 3439 (1994).

[129] S. Braunstein, C. Caves, and G. Milburn, Ann. Phys. 247, 135 (1996).

[130] X. B. Wang, T. Hisoshima, A. Tomita, and M. Hayashi, Phys. Rep. 448, 1 (2007).

[131] S. Amari and H. Nagaoka, Methods of Information Geometry (AMS \& Oxford University Press, Oxford, 2000).

[132] D. Petz, Linear Alg. Appl. 244, 81 (1996).

[133] J. Fiurasek and N. J. Cerf, Phys. Rev. Lett. 93, 063601 (2004). 